\title{
RECONSTRUCTING FUNCTION FIELDS FROM MILNOR K-THEORY
}

\author{
ANNA CADORET AND ALENA PIRUTKA
}

\begin{abstract}
Let $F$ be a finitely generated regular field extension of transcendence degree $\geq 2$ over a perfect field $k$. We show that the multiplicative group $F^{\times} / k^{\times}$endowed with the equivalence relation induced by algebraic dependence on $k$ determines the isomorphism class of $F$ in a functorial way. As a special case of this result, we obtain that the isomorphism class of the graded Milnor $K$-ring $K_{*}^{M}(F)$ determines the isomorphism class of $F$, when $k$ is algebraically closed or finite.
\end{abstract}

\section{INTRODUCTION}

This paper is motivated by the following general question, for which no counter-example seems to be known.

Question 1. Does the Milnor K-ring $K_{*}^{M}(F)$ determine the isomorphism class of the field $F$ ?

One may also ask whether or not the above holds in a functorial way, that is, whether or not the Milnor K-ring functor is fully faithfull from the groupoid of fields to the groupoid of $\mathbb{Z}_{>0}$-graded rings. This naive functorial version does not hold in general as shown by the example of finite fields $F$ where $K_{*}^{M}(F)$ has extra automorphisms induced by $x \rightarrow x^{u}$ for $u$ prime to $|F|-1$ on $K_{1}^{M}(F)$. To get a viable functorial version of Question 1, one should at least kill such extra automorphisms.

Since $K_{1}^{M}(F)=F^{\times}$, Question 1 essentially reduces to reconstructing the additive structure of $F$ from the multiplicative group $F^{\times}$endowed with additional data that can be detected by the Milnor K-ring. Our main result (Theorem 4) asserts that for a finitely generated regular field extension $F$ of transcendence degree $\geq 2$ over a perfect field $k$, the multiplicative group $F^{\times} / k^{\times}$endowed with the equivalence relation induced by algebraic dependence on $k$ determines the isomorphism class of $F$ in a functorial way. In Section 2, we show (Theorem 7) that for a finitely generated regular field extension of a field $k$ which is either algebraically closed or finite, the Milnor K-ring detects algebraic dependence. This is a consequence of deep K-theoretic results - the $n=2$ case of the Bloch-Kato conjecture [12] when $k$ is algebraically closed and of the Bass-Tate conjecture [15] when $k$ is finite. Combined with Theorem 4, this enables us show that the Milnor K-ring modulo the ideal of divisible elements (resp. of torsion elements) determines in a functorial way finitely generated regular field extensions of transcendence degree $\geq 2$ over algebraically closed fields (resp. over finite fields) (see Corollary 10). In particular, this provides a purely K-theoretic description of the group of birational automorphisms of normal projective varieties of dimension $\geq 2$ over algebraically closed or finite fields (Corollary 11).

Date: August 16, 2018. 
1.1. Main Result. Recall that a field extension $F / k$ is regular if $k$ is algebraically closed in $F$. Let $F / k$ be a regular field extension.

Definition 2. We say that $\bar{x}, \bar{y} \in F^{\times} / k^{\times}$are algebraically dependent and write $\bar{x} \equiv \bar{y}$ if either $\bar{x}=1=\bar{y}$ or $\bar{x} \neq 1 \neq \bar{y}$ and some (equivalently, all) lifts $x, y \in F^{\times}$of $\bar{x}, \bar{y} \in F^{\times} / k^{\times}$are algebraically dependent over $k$. The relation $\equiv$ is an equivalence relation on $F^{\times} / k^{\times}$.

Let $F / k, F^{\prime} / k^{\prime}$ be regular field extensions.

Definition 3. We say that a group morphism $\bar{\psi}: F^{\times} / k^{\times} \rightarrow F^{\prime \times} / k^{\prime \times}$ preserves algebraic dependence if for every $\bar{x}, \bar{y} \in F^{\times} / k^{\times}$the following holds: $\bar{x} \equiv \bar{y}$ if and only if $\bar{\psi}(\bar{x}) \equiv \bar{\psi}(\bar{y})$.

(In particular, a group morphism preserving algebraic dependence is automatically injective).

For a subfield $E \subset F$, write $\overline{E^{F}} \subset F$ for the algebraic closure of $E$ in $F$. Then a group morphism $\bar{\psi}: F^{\times} / k^{\times} \rightarrow F^{\prime \times} / k^{\prime \times}$ preserves algebraic dependence if and only if $\overline{k(x)^{F}} \times k^{\times}=$ $\bar{\psi}^{-1}\left(\overline{k^{\prime}(\psi(x))^{F^{\prime}}} / k^{\prime \times}\right)$ for every $x \in F$ and some (equivalently, every) lift $\psi(x) \in F^{\prime}$ of $\bar{\psi}(\bar{x})$.

Let $\operatorname{Isom}\left(F, F^{\prime}\right)$ denote the set of field isomorphisms $F \stackrel{\sim}{\rightarrow} F^{\prime}$ and

$$
\operatorname{Isom}\left(F / k, F^{\prime} / k^{\prime}\right) \subset \operatorname{Isom}\left(F, F^{\prime}\right)
$$

denote the subset of isomorphisms $F \stackrel{\sim}{\rightarrow} F^{\prime}$ inducing field isomorphisms $k \stackrel{\sim}{\rightarrow} k^{\prime}$.

Let $\operatorname{Isom}\left(F^{\times} / k^{\times}, F^{\prime \times} / k^{\prime \times}\right)$ denote the set of group isomorphisms $F^{\times} / k^{\times} \tilde{\rightarrow} F^{\prime \times} / k^{\prime \times}$ and

$$
\operatorname{Isom} \equiv\left(F^{\times} / k^{\times}, F^{\prime \times} / k^{\prime \times}\right) \subset \operatorname{Isom}\left(F^{\times} / k^{\times}, F^{\prime \times} / k^{\prime \times}\right)
$$

the subset of isomorphisms $F^{\times} / k^{\times} \stackrel{\sim}{\rightarrow} F^{\prime \times} / k^{\prime \times}$ preserving algebraic dependence. The group $\mathbb{Z} / 2$ acts on the set Isom $\equiv\left(F^{\times} / k^{\times}, F^{\prime \times} / k^{\prime \times}\right)$ by $\bar{\psi} \rightarrow \bar{\psi}^{-1}$. Write

$$
\overline{\mathrm{Isom}} \equiv\left(F^{\times} / k^{\times}, F^{\prime \times} / k^{\prime \times}\right)
$$

for the resulting quotient.

Theorem 4. Let $k, k^{\prime}$ be perfect fields of characteristic $p \geq 0$ and let $F / k, F^{\prime} / k^{\prime}$ be finitely generated regular field extensions of transcendence degree $\geq 2$. Then the canonical map

$$
\operatorname{Isom}\left(F / k, F^{\prime} / k^{\prime}\right) \rightarrow \overline{\operatorname{Isom}} \equiv\left(F^{\times} / k^{\times}, F^{\prime \times} / k^{\prime \times}\right)
$$

is bijective.

1.2. Comparison with existing results. Question 1 was considered by Bogomolov and Tschinkel in [3], where they prove (a variant of) Theorem 4 for finitely generated regular extensions of characteristic 0 fields ([3, Thm. 2]) and deduce from it Corollary 10 for finitely generated field extensions of algebraically closed fields of characteristic 0 ([3, Thm. 4]).

Variants of our results were also obtained by Topaz from a smaller amount of $K$-theoretic information - mod- $\ell$ Milnor $K$-rings (for finitely generated field extensions of transcendence degree $\geq 5$ over algebraically closed field of characteristic $p \neq \ell$ [16, Thm. B]) and rational Milnor $K$-rings (for finitely generated field extensions of transcendence degree $\geq 2$ over algebraically closed field of characteristic 0 [17, Thm. 6.1]) but enriched with the additional data of the so-called "rational quotients" of $F / k$. See also [17, Rem. 6.2] for some cases where the additional data of rational 
quotients can be removed.

Our strategy follows the one of Bogomolov and Tschinkel in [3], where the key idea is to parametrize lines in $F^{\times} / k^{\times}$as intersections of multiplicatively shifted (infinite dimensional) projective subspaces of a specific form arising from relatively algebraically closed subextensions of transcendence degree 1. See Subsection 1.3 for details. The strategy of Topaz is more sophisticated and goes through the reconstruction of the quasi-divisorial valuations of $F$ via avatars of the theory of commuting-liftable pairs as developped in the framework of birational anabelian geometry. Though not explicitly stated in the literature, it is likely that Theorem 4 and Corollary 10 for finitely generated field extensions algebraically closed fields of characteristic $p>0$ could also be recovered from the techniques of birational anabelian geometry as developed by Bogomolov-Tschinkel [4], Pop (e.g. [14], [13]) and Topaz.

To our knowledge, Theorem 4 for finitely generated regular extensions of perfect fields of characteristic $p>0$ and Corollary 10 for finitely generated field extensions of finite fields are new.

1.3. Strategy of proof. For simplicity, write $F^{p} \subset F$ for the subfield generated by $k$ and the $x^{p}$, $x \in F$ and $F^{\times} / p:=F^{\times} / F^{p \times}$.

The proof of Theorem 4 is carried out in Section 3. According to the fundamental theorem of projective geometry (see Lemma 29, for which we give a self-contained proof in the setting of possibly infinite field extensions), it would be enough to show that a group isomorphism $\bar{\psi}: F^{\times} / k^{\times} \stackrel{\rightarrow}{\rightarrow} F^{\prime \times} / k^{\prime \times}$ preserving algebraic dependence induces a bijection from lines in $F^{\times} / k^{\times}$to lines in $F^{\prime \times} / k^{\prime \times}$. This would reduce the problem to describing lines in $F^{\times} / k^{\times}$using only $\equiv$ and the multiplicative structure of $F^{\times} / k^{\times}$. This classical approach works well if $p=0$. The key observation of Bogomolov and Tschinkel in [3] is that every line can be multiplicatively shifted to a line passing through a "good" pair of points and that those lines can be uniquely parametrized as intersections of multiplicatively shifted (infinite dimensional) projective subspaces of a specific form arising from relatively algebraically closed subextensions of transcendence degree 1 [3, Thm. 22]. This is the output of elaborate computations in [3]. Later, Rovinsky suggested an alternative argument using differential forms; this is sketched in [4, Prop. 9].

When $p>0$, the situation is more involved. The original computations of [3] fail due to inseparability phenomena. Instead, we adjust the notion of "good" for the pair of points (Definition 18) in order to refine the argument of Rovinsky. In particular, we use the field-theoretic notion of "regular" element rather than the group-theoretic notion of "primitive" element used in [3]. To show that every line can be shifted to a line whose image contains a "good" pair of points (Lemma 19), one can invoke Bertini theorems ([7, Cor. 6.11.3] when $k$ is infinite and [5, Thm. 1.6] when $k$ is finite); we also give an alternative, more elementary argument due to Akio Tamagawa in Remark 21. This reduces the problem to show that $\bar{\psi}$ (or $\bar{\psi}^{-1}$ ) maps every line in $F^{\times} / k^{\times}$whose image contains a good pair of points $\left(\bar{x}_{1}, \bar{x}_{2}\right)$ isomorphically to a line in $F^{\prime \times} / k^{\prime} \times$. Actually, we cannot prove this directly when $p>0$. The issue is that, when $p>0$, the Bogomolov-Tschinkel parametrization of such line by the set $\mathfrak{I}\left(\bar{x}_{1}, \bar{x}_{2}\right)$ introduced in Subsection 3.1 is much rougher than in [3, Thm. 22] - up to prime-to- $p$ powers and certain affine transformations with $F^{p}$-coefficients (Lemma 22); this is due to the apparition of constants in $F^{p}$ when one integrates differentials forms. Lemma 22 is 
however enough to show that there exists a unique $m \in \mathbb{Z}$ normalized as

$$
\begin{array}{ll}
|m|=1 & \text { if } p=0,2 \\
1 \leq|m| \leq \frac{p-1}{2} & \text { if } p>2 ;
\end{array}
$$

such that $\bar{\psi}^{m}$ induces a bijection from lines in $F^{\times} / p$ to lines in $F^{\prime \times} / p$ (Proposition 26). So that Lemma 29 gives a unique field isomorphism $\phi: F \stackrel{\sim}{\rightarrow} F^{\prime}$ such that the resulting isomorphism of groups $\phi: F^{\times} \tilde{\rightarrow} F^{\prime \times}$ coincides with $\bar{\psi}^{m}$ on $F^{\times} / p$. This concludes the proof if $p=0$. But if $p>0$, the extension $F / F^{p}$ is much smaller (finite-dimensional!) and one has to perform an additional descent step (Section 3.6) to show that $m= \pm 1$ and $\phi$ coincides with $\bar{\psi}^{ \pm 1}$ on $F^{\times} / k^{\times}$(not only on $\left.F^{\times} / p\right)$.

We limited our exposition to function fields, which are those of central interest in algebraic geometry. However, some of our results extend to more exotic fields provided they behave like function fields. For instance, Theorem 4 works for the class of regular field extensions $F$ of transcendence degree $\geq 2$ over a perfect field $k$ such that for every subfield $k \subset E \subset F$ of transcendence degree 2 over $k$, the algebraic closure of $E$ in $F$ is a finite extension of $E$. We do not elaborate on this.

1.4. Acknowledgements. The first author was partially supported by the I.U.F. and the ANR grant ANR-15-CE40-0002-01. The second author was partially supported by NSF grant DMS1601680 and by the Laboratory of Mirror Symmetry NRU HSE, RF Government grant, ag. no. 14.641.31.0001. This project was initiated at the occasion of a working group on geometric birational anabelian geometry at the Courant Institute of Mathematics, NYU. The authors would like to thank F. Bogomolov and Y. Tschinkel for inspiring exchanges, B. Kahn for his explanations concerning the Bass-Tate conjecture and A. Merkurjev, M. Morrow and A. Tamagawa for helpful remarks on a first version of this text. They also thank A. Tamagawa for suggesting the more elementary proof of Lemma 19 presented in Remark 21.

\section{Milnor K-RIngs AND Algebraic DePendence}

Let $K_{*}^{M}(-)$ denote the Milnor K-ring functor, from the groupoid $\mathcal{F}$ of fields to the groupoid $\mathcal{A}$ of associative $\mathbb{Z}_{\geq 0}$-graded anti-commutative rings.

For $p=0$ or a prime, let $\mathcal{F}_{p} \subset \mathcal{F}$ denote the full subgroupoid of fields of characteristic $p$.

2.1. Some geometric observations. Let $F / k$ be a finitely generated regular field extension.

2.1.1. Let $x_{1}, \ldots, x_{n} \in F$ be such that $k\left(x_{1}, \ldots, x_{n}\right)$ has transcendence degree $n$ over $k$. Then

Lemma 5. There exists a divisorial valuation $v$ of $F$ such that

- $v\left(x_{1}\right) \neq 0, v\left(x_{2}\right)=\cdots=v\left(x_{n}\right)=0$;

- the images $\bar{x}_{2}, \ldots, \bar{x}_{n}$ of $x_{2}, \ldots, x_{n}$ in the residue field $k(v)$ are algebraically independent over $k$.

In particular, one can compute explicitly the image of $\left\langle x_{1}, \ldots, x_{n}\right\rangle$ via the residue map $\partial_{v}^{n}$ : $K_{n}^{M}(F) \rightarrow K_{n-1}^{M}(k(v))$ as

$$
\partial_{v}^{n}\left(\left\langle x_{1}, \ldots, x_{n}\right\rangle\right)= \pm v\left(x_{1}\right)\left\langle\bar{x}_{2}, \ldots, \bar{x}_{n}\right\rangle= \pm\left\langle\bar{x}_{2}^{v\left(x_{1}\right)}, \bar{x}_{3}, \ldots, \bar{x}_{n}\right\rangle .
$$

Proof. Fix a normal projective model $X / k$ of $F / k$. Each $x_{i}$ defines a dominant rational function $x_{i}: X \rightarrow \mathbb{P}_{k}^{1}$ such that

$$
\underline{x}=\left(x_{1}, \ldots, x_{n}\right): X \rightarrow\left(\mathbb{P}_{k}^{1}\right)^{n}
$$

is again dominant. Choose any open subscheme $U \subset X$ over which the map $\underline{x}: U \rightarrow\left(\mathbb{P}_{k}^{1}\right)^{n}$, $i=1, \ldots, n$ is defined. Then, up to replacing $X$ by the normalization of the Zariski closure of the 
graph of $\left.\underline{x}\right|_{U}$ in $X \times\left(\mathbb{P}_{k}^{1}\right)^{n}$, one may assume that the maps $x_{i}, i=1, \ldots, n$ and $\underline{x}$ are defined over $X$, and surjective. Choose an irreducible divisor $D \in \operatorname{Div}(X)$ with $v_{D}\left(x_{1}\right) \neq 0$. Then (since $\underline{x}(D)$ has codimension at most 1 in $\left.\left(\mathbb{P}_{k}^{1}\right)^{n}\right)$ the restriction $\left.\underline{x}\right|_{D}: D \rightarrow\left(\mathbb{P}_{k}^{1}\right)^{n}$ surjects onto $0 \times\left(\mathbb{P}_{k}^{1}\right)^{n-1} \subset\left(\mathbb{P}_{k}^{1}\right)^{n}$; in particular the elements $\bar{x}_{i}:=\left.x_{i}\right|_{D} \in k(D), i=2, \ldots, n$ remain algebraically independent over $k$ and $v_{D}\left(x_{i}\right)=0, i=2, \ldots, n$.

2.1.2. The abelian group $F^{\times} / k^{\times}$is a free abelian group since it embeds into a free abelian group. This follows from the exact sequence

$$
0 \rightarrow k^{\times} \rightarrow F^{\times} \rightarrow \operatorname{Div}(X),
$$

where $X / k$ is any normal projective model $X / k$ of $F / k$, and the fact that $\operatorname{Div}(X)$ is a free abelian group.

2.2. Type. We say that a field $F$ is a function field over a field $k$ (or with field of constants $k$ ) if $F$ is a finitely generated regular field extension of transcendence degree $\geq 1$ over $k$. We say that a function field $F$ over $k$ is of type 1 (resp. of type 2) if $k$ is algebraically closed (resp. finite), in which case $k$ is uniquely determined by $F$ (see Lemma 6 below).

Let $\widetilde{\mathcal{F}} \subset \mathcal{F}, \widetilde{\mathcal{F}}_{p} \subset \mathcal{F}_{p}$ denote the full subcategories of function fields of type 1,2 .

Lemma 6. For $F \in \widetilde{\mathcal{F}}$, the type, the multiplicative group $k^{\times}$of the field of constants and the characteristic $p$ of $F$ are determined by $F^{\times}=K_{1}^{M}(F)$ as follows.

\begin{tabular}{|c|c|c|c|}
\hline Type & $\begin{array}{c}\text { Torsion subgroup } \\
\text { of } F^{\times}\end{array}$ & $k^{\times}$ & $p$ \\
\hline 1 & $\begin{array}{l}\text { Infinite } \\
\text { Finite }\end{array}$ & $\begin{array}{l}\text { Divisible } \\
\text { subgroup of } F^{\times} \\
\text {Torsion } \\
\text { subgroup of } F^{\times}\end{array}$ & $\begin{array}{c}0 \text { or unique } p \text { such that } \\
p \cdot: k^{\times} \rightarrow k^{\times} \text {is an isomorphism } \\
\text { Unique } p \text { such that } \log \left(\left|k^{\times}\right|+1\right) \in \mathbb{Z} \log (p)\end{array}$ \\
\hline
\end{tabular}

In the following, we sometimes write $F / k \in \widetilde{\mathcal{F}}$ instead of $F \in \widetilde{\mathcal{F}}$ implicitly meaning that $k$ is the field of constants of $F$.

2.3. Detecting algebraic dependence. For $F \in \mathcal{F}_{p}$ and a prime $\ell \neq p$, let $D K_{*}^{M}(F) \subset K_{*}^{M}(F)$ (resp. $T K_{*}^{M}(F) \subset K_{*}^{M}(F)$ ) denote the (two-sided) ideal of elements which are infinitely $\ell$-divisible (resp. torsion) with respect to the structure of $\mathbb{Z}$-module and write

$$
\begin{aligned}
& \bar{K}_{*}^{1, M}(F):=K_{*}^{M}(F) / D K_{*}^{M}(F) ; \\
& \bar{K}_{*}^{2, M}(F):=K_{*}^{M}(F) / T K_{*}^{M}(F) .
\end{aligned}
$$

Then $K_{*}^{M} \rightarrow \bar{K}_{*}^{i, M}$ is a morphism of functors from $\mathcal{F}$ to $\mathcal{A}, i=1,2$.

Theorem 7. For $F \in \widetilde{\mathcal{F}}_{p}$ of type $i$ and every $x_{1}, \ldots, x_{n} \in F^{\times}$consider the following assertions.

(i) $\left\langle x_{1}, \ldots, x_{n}\right\rangle=0$ in $\bar{K}_{n}^{i, M}(F)$;

(ii) the transcendence degree of $k\left(x_{1}, \ldots, x_{n}\right)$ over $k$ is $\leq n-1$.

Then, (i) $\Rightarrow$ (ii) and (ii) $\Rightarrow$ (i) if $i=1$ or if $i=2$ and $n \leq 2$. 
Proof. The assertion for $n=1$ follows from 2.1.2.

(i) $\Rightarrow$ (ii): We proceed by induction on $n$. Assume $k\left(x_{1}, \ldots, x_{n}\right)$ has transcendence degree $n$ over $k$ and choose a place $v$ of $F$ as in Lemma 5. By induction hypothesis $\partial_{v}^{n}\left(\left\langle x_{1}, \ldots, x_{n}\right\rangle\right) \neq 0$ in $\bar{K}_{n-1}^{i, M}(k(v))$ hence, a fortiori, $\left\langle x_{1}, \ldots, x_{n}\right\rangle \neq 0$ in $\bar{K}_{n}^{i, M}(F)$.

(ii) $\Rightarrow$ (i): Assume $E=k\left(x_{1}, \ldots, x_{n}\right)$ has transcendence degree $d \leq n-1$ over $k$. Since $\left\langle x_{1}, \ldots, x_{n}\right\rangle$ lies in the image of the restriction map $\bar{K}_{n}^{i, M}(E) \rightarrow \bar{K}_{n}^{i, M}(F)$, it is enough to show that $\bar{K}_{n}^{i, M}(E)=0$. If $F$ is of type 1, this follows from Tsen's theorem [9], which ensures that $H^{n}\left(E, \mathbb{Z}_{\ell}(n)\right)=0$ and from the Bloch-Kato conjecture $[18,19]$. If $F$ is of type 2 , this follows from the $n=2$ case of the Bass-Tate conjecture [15, Thm. 1].

Let $F, F^{\prime} \in \widetilde{\mathcal{F}}_{p}$ of the same type $i$. For a morphism $\bar{\psi}_{*}: \bar{K}_{*}^{i, M}(F) \rightarrow \bar{K}_{*}^{i, M}\left(F^{\prime}\right)$ of $\mathbb{Z}_{\geq 0}$-graded rings and integer $n \geq 1$, consider the assertion

$$
(\circ, n) \text { For every } x_{1}, \ldots, x_{n} \in F, \bar{\psi}_{1}\left(\overline{k\left(x_{1}, \ldots, x_{n}\right)^{F}} \times / k^{\times}\right) \circ \overline{k^{\prime}\left(\psi_{1}\left(x_{1}\right), \ldots, \psi_{1}\left(x_{n}\right)\right)^{F^{\prime}}} / k^{\prime} \times
$$

where $\psi_{1}: F^{\times} \rightarrow F^{\prime} \times$ denotes any set-theoretic lift of $\bar{\psi}_{1}$ and $\circ$ means the symbol $=$ or $\subset$.

Corollary 8. Then Assertion $(\subset, n)$ holds for every $n$ if $i=1$ and for $n \leq 2$ if $i=2$. If $\bar{\psi}_{*}$ is an isomorphism of $\mathbb{Z}_{\geq 0}$-graded rings, Assertion $(=, n)$ holds for every $n$ if $i=1$ and for $n \leq 2$ if $i=2$.

\section{Remark 9.}

- When $F$ is of type 2 the implication (ii) $\Rightarrow$ (i) of Theorem 7 and the assertions $(\subset, n),(=, n)$ of Corollary 8 for every $n$ are predicted by the Bass-Tate conjecture in positive characteristic [2, Question, p.390]. See also [8] for the relation between the Bass-Tate conjecture in positive characteristic and classical motivic conjectures.

- For our applications, we only need the $n \leq 2$ cases of Corollary 8. In particular, for function fields of type 1 , we only need the $n=2$ case of the Bloch-Kato conjecture, an earlier theorem of Merkurjev and Suslin [12].

2.4. Reconstructing function fields. The $n \leq 2$ case of Corollary 8 implies that for $i=1,2$ and for every $F, F^{\prime} \in \widetilde{\mathcal{F}}_{p}$ of type $i$ and isomorphism $\bar{\psi}_{*}: \bar{K}_{*}^{i, M}(F) \rightarrow \bar{K}_{*}^{i, M}\left(F^{\prime}\right)$ of $\mathbb{Z}_{\geq 0}$-graded rings the induced isomorphism of multiplicative groups $\bar{\psi}_{1}$ preserves algebraic dependence. This implies the following. For $i=1,2$, let

$$
\operatorname{Isom}\left(\bar{K}_{*}^{i, M}(F), \bar{K}_{*}^{M}\left(F^{\prime}\right)\right)
$$

denote the set of isomorphisms of $\mathbb{Z}$-graded rings $\bar{K}_{*}^{i, M}(F) \sim \bar{K}_{*}^{i, M}\left(F^{\prime}\right)$ and

$$
\overline{\operatorname{Isom}}\left(\bar{K}_{*}^{i, M}(F), \bar{K}_{*}^{i, M}\left(F^{\prime}\right)\right)
$$

their quotients by the natural action of $\mathbb{Z} / 2$.

Corollary 10. Let $F, F^{\prime} \in \tilde{\mathcal{F}}$. Then

(i) $\operatorname{Isom}\left(K_{*}^{M}(F), K_{*}^{M}\left(F^{\prime}\right)\right) \neq \emptyset$ only if $F, F^{\prime}$ are of the same type $i$ and characteristic $p$.

(ii) If $F, F^{\prime} \in \tilde{\mathcal{F}}_{p}$ are of the same type $i$ and have transcendence degree $\geq 2$ over their fields of constants then the natural functorial map

$$
\operatorname{Isom}\left(F, F^{\prime}\right) \rightarrow \overline{\operatorname{Isom}}\left(\bar{K}_{*}^{i, M}(F), \bar{K}_{*}^{i, M}\left(F^{\prime}\right)\right)
$$

is bijective. 
Proof. Part (i) follows from Lemma 6. For Part (ii), one has a canonical commutative diagram

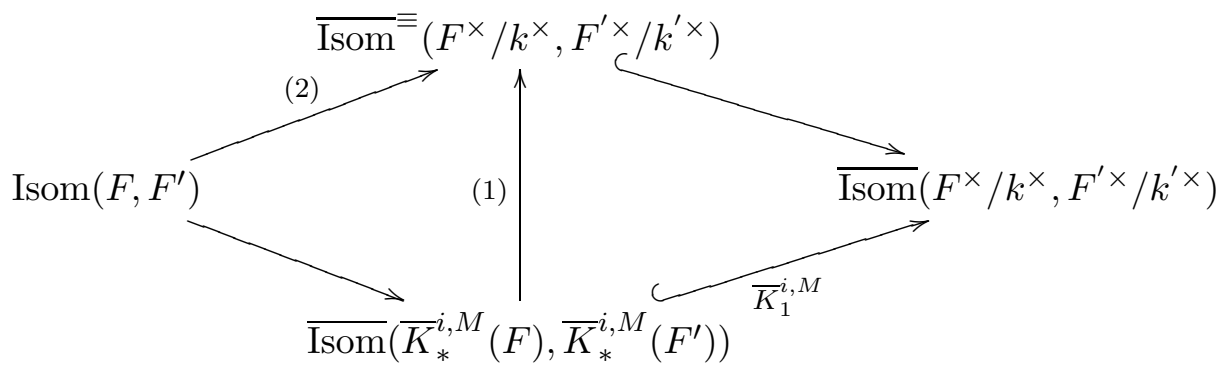

where the factorization (1) follows from Corollary 8. So the conclusion follows from the fact that the map (2) is bijective by Theorem 4 .

Corollary 11. Let $F \in \tilde{\mathcal{F}}$ of type $i$. Then one has the following group isomorphisms

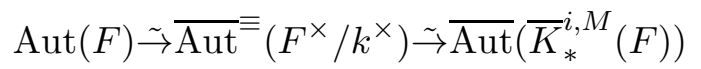

Remark 12. For convenience, we stated Corollary 10 with the whole rings $\bar{K}_{*}^{i, M}(F)$ but, it is actually enough to consider the datum of $\bar{K}_{\leq 2}^{i, M}(F)$ that is the canonical pairing

$$
\langle-,-\rangle: \bar{K}_{1}^{i, M}(F) \otimes \bar{K}_{1}^{i, M}(F) \rightarrow \bar{K}_{2}^{i, M}(F),
$$

(for $i=1$, one can even replace $\bar{K}_{2}^{1, M}(F)$ with its $\ell$-adic completion $H^{2}\left(F, \mathbb{Z}_{\ell}(2)\right)$ ).

Remark 13. From Lemma 6, one can reconstruct the field of constants $k$ of a function field $F$ in $\tilde{\mathcal{F}}$ from $K_{1}^{M}(F)$. In general, one may ask for a relative version of Corollary 10 that is replacing the functor $K_{*}^{M}(-)$ with the functor sending a finitely generated field extension $F$ of a perfect field $k$ to the morphism $K_{*}^{M}(k) \rightarrow K_{*}^{M}(F)$.

The remaining part of the paper is devoted to the proof of Theorem 4 .

\section{Proof of Theorem 4}

We refer to Subsection 1.3 for a description of the strategy of the proof.

\subsection{Notation.}

Let $k$ be a perfect field of characteristic $p \geq 0$ and let $F / k$ be a finitely generated regular field extension of transcendence degree $\geq 2$.

For every subfield $k \subset E \subset F$ and $\bar{x} \neq \bar{y} \in F^{\times} / E^{\times}$, write

$$
\mathfrak{l}_{E}(\bar{x}, \bar{y})=(E x \oplus E y)^{\times} / E^{\times} \subset F^{\times} / E^{\times}
$$

for the corresponding line in $F^{\times} / E^{\times}$.

Definition 14. We say that $\bar{x}, \bar{y} \in F^{\times} / k^{\times}$are $p$-multiplicatively dependent and write $\bar{x} \sim_{p} \bar{y}$ if

$$
\bar{x}^{\mathbb{Z}} \cap \bar{y}^{\mathbb{Z}} \neq 1
$$

in $F^{\times} / p$. The relation $\sim_{p}$ is an equivalence relation on $F^{\times} / k^{\times}$. 
For $\bar{x}_{1}, \bar{x}_{2}, \bar{y}_{1}, \bar{y}_{2} \in F^{\times} / k^{\times}$and some (equivalently, every) lifts $x_{1}, x_{2}, y_{1}, y_{2} \in F^{\times}$, write

$$
\mathcal{I}\left(\bar{x}_{1}, \bar{x}_{2}, \bar{y}_{1}, \bar{y}_{2}\right):=\left(\overline{k\left(x_{1} / x_{2}\right)^{F}} \times x_{2}\right) \bigcap\left(\overline{k\left(y_{1} / y_{2}\right)^{F}} \times y_{2}\right)
$$

and

$$
\mathfrak{I}\left(\bar{x}_{1}, \bar{x}_{2}\right)=\bigcup_{y_{1}, y_{2}} \mathcal{I}\left(\bar{x}_{1}, \bar{x}_{2}, \bar{y}_{1}, \bar{y}_{2}\right)
$$

where the union is over all $y_{i} \in{\overline{k\left(x_{i}\right)^{F}}}^{\times}, x_{i} \chi_{p} y_{i}, i=1,2$.

Let $\overline{\mathcal{I}}\left(\bar{x}_{1}, \bar{x}_{2}, \bar{y}_{1}, \bar{y}_{2}\right), \overline{\mathfrak{I}}\left(\bar{x}_{1}, \bar{x}_{2}\right)$ denote the images of $\mathcal{I}\left(\bar{x}_{1}, \bar{x}_{2}, \bar{y}_{1}, \bar{y}_{2}\right), \mathfrak{I}\left(\bar{x}_{1}, \bar{x}_{2}\right)$ in $F^{\times} / k^{\times}$respectively.

For every $\bar{I} \in \overline{\mathfrak{I}}\left(\bar{x}_{1}, \bar{x}_{2}\right)$, let $\mathfrak{l}^{\circ}\left(\bar{I}, \bar{x}_{1}\right)$ denote the set of all $y_{1} \in \overline{k\left(x_{1}\right)^{F}}, x_{1} \chi_{p} y_{1}$ such that for some (equivalently, every) lift $I \in F^{\times}$of $\bar{I} \in F^{\times} / k^{\times}$, one has $I \in{\overline{k\left(y_{1} / y_{2}\right)^{F}}}^{\times} \cdot y_{2}$ for some $y_{2} \in \overline{k\left(x_{2}\right)^{F}}$, $x_{2} \varkappa_{p} y_{2}$.

Similarly, let $\mathfrak{l}^{\circ}\left(\bar{I}, \bar{x}_{2}\right)$ denote the set of all $y_{2} \in \overline{k\left(x_{2}\right)^{F}}, x_{2} \chi_{p} y_{2}$ such that for some (equivalently, every) lift $I \in F^{\times}$of $\bar{I} \in F^{\times} / k^{\times}$, one has $I \in \overline{k\left(y_{1} / y_{2}\right)^{F}} \times y_{2}$ for some $y_{1} \in \overline{k\left(x_{1}\right)^{F}}, x_{1} \varkappa_{p} y_{1}$.

Set also

$$
\mathfrak{l}\left(\bar{I}, \bar{x}_{i}\right)=\mathfrak{l}^{\circ}\left(\bar{I}, \bar{x}_{i}\right) \cup\left\{x_{i}\right\}, i=1,2 .
$$

Let $\overline{\mathfrak{l}}^{\circ}\left(\bar{I}, \bar{x}_{i}\right), \overline{\mathfrak{l}}\left(\bar{I}, \bar{x}_{i}\right)$ denote the images of $\mathfrak{l}^{\circ}\left(\bar{I}, \bar{x}_{i}\right), \mathfrak{l}\left(\bar{I}, \bar{x}_{i}\right)$ in $F^{\times} / k^{\times}$respectively.

\subsection{Recollection on differentials.}

Lemma 15. For $x_{1}, \ldots, x_{n} \in F$, the following are equivalent

- $x_{1}, \ldots, x_{n} \in F$ is a separating transcendence basis for $F / k$, i.e. $F / k\left(x_{1}, \ldots, x_{n}\right)$ is a finite separable field extension;

- $d x_{1}, \ldots, d x_{n} \in \Omega_{F \mid k}^{1}$ is an $F$-basis of $\Omega_{F \mid k}^{1}$.

If $p>0$, these are also equivalent to

- $x_{1}, \ldots, x_{n} \in F$ is a p-basis of $F / k$ that is a $\mathbb{F}_{p}$-basis of $F / F^{p}$.

See e.g $[11, \S 27$, Thm 59; $\S 38$, Thm. 86] for the proof. Recall that since $k$ is a perfect field, the extension $F / k$ always admits a separating transcendence basis, and that if $p=0$, every transcendence basis is separating.

Corollary 16. $\operatorname{ker}\left(d: F \rightarrow \Omega_{F \mid k}^{1}\right)=F^{p}$.

In particular:

3.2.1. For every $x \in F$, the following are equivalent:

(i) $x \in F \backslash F^{p}$;

(ii) $d x \neq 0$;

(iii) $x$ is a separating transcendence basis for $\overline{k(x)^{F}} / k$. 
If $x \in F$ verifies the above equivalent conditions (i), (ii), (iii), for every $0 \neq f \in \overline{k(x)^{F}}$ there exists a unique $f^{\prime}:=f^{\prime}(x) \in \overline{k(x)^{F}}$ such that $d f=f^{\prime} d x$.

3.2.2. For every $x_{i} \in F, i=1,2$, with $d x_{1}, d x_{2} \in \Omega_{F \mid k}^{1}$ linearly independent over $F$, and $z_{i} \in$ $\overline{k\left(x_{i}\right)^{F}} \backslash k^{\times}, i=1,2$, the following are equivalent:

(i) $z_{1} / z_{2} \in F \backslash F^{p}$;

(ii) $d z_{1}, d z_{2}$ are linearly independent over $F$;

(iii) $z_{i}^{\prime} \neq 0, i=1,2$;

(iv) $z_{i} \in F \backslash F^{p}, i=1,2$.

\subsection{Shifting multiplicatively lines to lines passing through good pairs.}

Let $k$ be a perfect field of characteristic $p \geq 0$ and let $F / k$ be a finitely generated regular field extension of transcendence degree $\geq 2$.

Definition 17. We say that $x \in F$ is $F / k$-regular if $x$ is transcendental over $k$ and $F / k(x)$ is a regular field extension.

Definition 18. We say that $\left(\bar{x}_{1}, \bar{x}_{2}\right) \in F^{\times} / k^{\times}$is a good pair if for some (equivalently, every) lifts $x_{1}, x_{2} \in F^{\times}$of $\bar{x}_{1}, \bar{x}_{2} \in F^{\times} / k^{\times}, x_{1}$ is $F / k$-regular and $d x_{1}, d x_{2} \in \Omega_{F \mid k}^{1}$ are linearly independent over $F$.

Lemma 19. For every $x \in F^{\times} \backslash F^{\times p}$, there exists a $F / k$-regular $y \in F^{\times}$such that $d x$, $d y$ are linearly independent over $F$.

In particular, for every $\bar{x} \in F^{\times} / k^{\times}, \bar{x} \neq 1$ in $F^{\times} / p$ there exists $\bar{y} \in F^{\times} / k^{\times}$such that $(\bar{y}, \overline{x y}) \in$ $F^{\times} / k^{\times}$is a good pair.

Proof. This follows from Bertini theorems. Since $F$ has finite transcendence degree $r \geq 2$ over $k$ and $k$ is perfect, there exist $x_{2}, \ldots, x_{r} \in F^{\times}$such that $d x, d x_{2}, \ldots, d x_{r}$ are linearly independent over $F$. Write $E:=k\left(x_{2}, \ldots, x_{r}\right)$. Let $z \in F^{\times}$with minimal polynomial

$$
P_{z}=T^{d}+\sum_{0 \leq i \leq d-1} a_{i} T^{i} \in E(x)[T]
$$

over $E(x)$. Then $d x, d z$ are linearly dependent over $F$ if and only if $\partial a_{i} / \partial x_{j}=0, i=0, \ldots, d-1, j=$ $2, \ldots n$, or equivalently, $P_{z} \in E^{p}(x)[T]$. In particular, any $y \in E(x) \backslash E^{p}(x)$ will have the property that $d x, d y$ are linearly independent over $F$. We claim that one can find $y \in E(x) \backslash E^{p}(x)$ such that $y$ is $F / k$-regular. Fix a normal quasi-projective model $X / k$ of $F / k$ such that $x, x_{2}, \ldots, x_{r}$ induce a finite dominant morphism $\underline{x}: X \rightarrow \mathbb{P}_{k}^{r}$. It is enough to show there exists a homogeneous polynomial $P \in k\left[x, x_{1}, \ldots, x_{r}\right] \backslash k\left[x, x_{1}^{p}, \ldots, x_{r}^{p}\right]$ such that the fiber at 0 of the composite map

$$
y: X \stackrel{x}{\rightarrow} \mathbb{P}_{k}^{r} \stackrel{P}{\rightarrow} \mathbb{P}_{k}^{1}
$$

is geometrically irreducible. Viewing $y$ as an element in $E(x)$, we deduce that $y$ is $F / k$-regular, by $[6,9.7]$. If $k$ is infinite, this follows directly from [7, Cor. 6.11.3]. If $k$ is finite, this follows from [5, Thm. 1.6] and Lemma 20 below (note that $\frac{1}{p^{r-1}}<1$ since $r \geq 2$ ).

Set $S^{\prime}:=k\left[x, x_{2}^{p}, \ldots, x_{r}^{p}\right] \subset k\left[x, x_{2}, \ldots, x_{r}\right]$ and define the density of $S^{\prime}$ as

$$
\delta\left(S^{\prime}\right)=\lim _{d \rightarrow+\infty} \frac{\left|S^{\prime} \cap S_{d}\right|}{\left|S_{d}\right|},
$$

where $S_{d} \subset k\left[x, x_{2}, \ldots, x_{r}\right]$ denotes the set of homogeneous polynomials of degree $d$. Then 
Lemma 20. $\delta\left(S^{\prime}\right)=\frac{1}{p^{r-1}}$.

Proof. One has $\left|S_{d}\right|=\left(\begin{array}{c}d+r-1 \\ d\end{array}\right)$ and $\left|S^{\prime} \cap S_{d}\right|=\sum_{0 \leq n \leq\left\lfloor\frac{d}{p}\right\rfloor}\left(\begin{array}{c}n+r-2 \\ n\end{array}\right)$. To estimate $\frac{\left|S^{\prime} \cap S_{d}\right|}{\left|S_{d}\right|}$, write $a:=\left\lfloor\frac{d}{p}\right\rfloor, b:=d-p a$ and

$$
Q_{r}(T):=\prod_{1 \leq k \leq r-2}(T+k)-T^{r-2}=\sum_{0 \leq i \leq r-3} a_{i} T^{i} \in \mathbb{Z}[T]
$$

Then

$$
\frac{\left|S^{\prime} \cap S_{d}\right|}{\left|S_{d}\right|}=\left(\begin{array}{c}
d+r-1 \\
d
\end{array}\right)^{-1} \sum_{0 \leq n \leq a}\left(\begin{array}{c}
n+r-2 \\
n
\end{array}\right)=\frac{r-1}{\prod_{1 \leq k \leq r-1}\left(1+\frac{b+k}{p a}\right)} \frac{1}{(p a)^{r-1}} \sum_{0 \leq n \leq a} \prod_{1 \leq k \leq r-2}(n+k)
$$

with

$$
\sum_{0 \leq n \leq a} \prod_{1 \leq k \leq r-2}(n+k)=\sum_{0 \leq n \leq a} n^{r-2}+\sum_{0 \leq i \leq r-3} a_{i} \sum_{0 \leq n \leq a} n^{i}
$$

By Faulhaber's formula

$$
\sum_{1 \leq n \leq a} n^{i}=\frac{a^{i+1}}{i+1}+\frac{1}{2} a^{i}+\sum_{2 \leq k \leq i} \frac{B_{k}}{k !} \frac{i !}{(i-k+1) !} a^{i-k+1}
$$

(where the $B_{k}$ are the Bernoulli numbers) one gets

$$
\sum_{0 \leq n \leq a} \prod_{1 \leq k \leq r-2}(n+k) \sim \frac{a^{r-1}}{r-1}
$$

whence the assertion.

Remark 21. We resorted to Bertini theorems, which provide a conceptually natural proof of Lemma 19 but one can give more elementary arguments. If $k$ is infinite, the Galois-theoretic Lemma [10, Chap. VIII, Lem. in Proof of Thm. 7] already shows there exists (infinitely many) $0 \neq a \in k$ such that $y:=a x_{2}+x$ is $F / k$-regular; by construction $d x, d y$ are linearly independent over $F$. If $k$ is finite, Akio Tamagawa suggested the following arguments. Fix a smooth (not necessarily proper) model $X / k$ of $F / k$ and a closed point $t \in X$. Let $\widetilde{X} \rightarrow X$ denote the blow-up of $X$ at $t$ and $D_{t}=\mathbb{P}_{k(t)}^{n-1} \subset \tilde{X}$ the exceptional divisor. Fix a non-empty affine subset $U=\operatorname{Spec}(R) \subset \tilde{X}$ such that $Z:=U \cap D_{t}$ is non-empty and the rational map $x: \tilde{X} \rightarrow X \rightarrow \mathbb{A}_{k}^{1}$ given by $x$ is defined over $U$. We endow $Z$ with its reduced subscheme structure. Since $Z$ is irreducible, one can write $Z=\operatorname{Spec}(R / P)$ for some prime ideal $P$ in $R$. Pick $y \in R \backslash R \cap F^{p} \overline{k(x)^{F}}$ such that $f \bmod P \in R / P \backslash k(t)$. Let $\overline{k[y]^{F}} \subset F$ denote the normal closure of $k[y]$ in $\overline{k(y)^{F}} / k(y)$. Since $R$ is smooth over $k$ hence normal, $\overline{k[y]^{F}} \subset R$. Also, by our choice of $y$, the morphism $\overline{k[y]^{F}} \hookrightarrow R \rightarrow R / I$ is injective whence the fraction field $\overline{k(y)^{F}}$ of $\overline{k[y]^{F}}$, embeds into the fraction field $k(t)\left(x_{2}, \ldots, x_{n}\right)$ of $R / I$. Since $\overline{k(y)^{F}} / k$ is regular, $k(t) \otimes_{k} \overline{k(y)^{F}} \simeq k(t) \cdot \overline{k(y)^{F}} \subset k(t)\left(x_{2}, \ldots, x_{n}\right)$. From Luröth theorem, one thus has $k(t) \otimes_{k} \overline{k(y)^{F}}=k(t)(T)$ for some $T \in k(t)\left(x_{2}, \ldots, x_{n}\right)$ transcendent over $k(t)$. In other words, $\overline{k(y)^{F}}$ is the function field of a $k(t) / k$ form $C_{y}$ of $\mathbb{P}_{k}^{1}$. Since $k$ is finite hence perfect with trivial Brauer group, $C_{y} \simeq \mathbb{P}_{k}^{1}$. This shows $y \in F$ is $F / k$ regular. Since $y \notin F^{p} \overline{k(x)^{F}}$, $d x, d y$ are linearly independent over $F$. 


\subsection{Approximating lines passing through good pairs up to powers.}

Let $k$ be a perfect field of characteristic $p \geq 0$ and let $F / k$ be a finitely generated regular field extension of transcendence degree $\geq 2$.

Lemma 22. Let $\left(\bar{x}_{1}, \overline{x_{2}}\right) \in F^{\times} / k^{\times}$be a good pair. Then, for every $\bar{I} \in \bar{\Im}\left(\bar{x}_{1}, \bar{x}_{2}\right)$ there exists $m \in \mathbb{Z}$ satisfying (1.1), $N \in \mathbb{Z}, \alpha=\alpha\left(\frac{x_{1}}{x_{2}}\right) \in{\overline{k\left(\frac{x_{1}}{x_{2}}\right)^{F}}}^{\times}$and $c \in k^{\times}$such that

$$
I^{m}=\alpha\left(\frac{x_{1}}{x_{2}}\right)^{p}\left(x_{1}^{m}-c \frac{x_{1}^{p N}}{x_{2}^{p N}} x_{2}^{m}\right) \in \mathfrak{l}_{F^{p}}\left(x_{1}^{m}, x_{2}^{m}\right) .
$$

Furthermore, for every $y_{i} \in \overline{k\left(x_{i}\right)^{F}}, y_{i} \varkappa_{p} x_{i}, i=1,2$ such that $I \in \mathcal{I}\left(\bar{x}_{1}, \bar{x}_{2}, \bar{y}_{1}, \bar{y}_{2}\right)$, one has for $i=1,2$

$$
y_{i}^{m}=\alpha_{i}^{p}\left(x_{i}^{m}-c_{i} x_{i}^{p N}\right)
$$

for some $\alpha_{i} \in \overline{k\left(x_{i}\right)^{F}}, c_{i} \in k^{\times}$with the condition $c=c_{1} / c_{2}$.

Proof. The proof when $p=0$ is significantly simpler since $\operatorname{ker}(d)=k$ (recall $F / k$ is regular). We carry out the proof for $p>0$ and just mention the simplifications that occur for $p=0$. The results for $p=0$ are exactly similar but with elements in $F^{p}$ replaced by elements in $k$.

We are to determine the possible $y_{i} \in{\overline{k\left(x_{i}\right)^{F}}}^{\times}, y_{i} \chi_{p} x_{i}, i=1,2$ such that $\mathcal{I}\left(\bar{x}_{1}, \bar{x}_{2}, \bar{y}_{1}, \bar{y}_{2}\right) \neq \emptyset$ and for all such $y_{i}, i=1,2$ the elements $I \in \mathcal{I}\left(\bar{x}_{1}, \bar{x}_{2}, \bar{y}_{1}, \bar{y}_{2}\right)$. So assume $\mathcal{I}\left(\bar{x}_{1}, \bar{x}_{2}, \bar{y}_{1}, \bar{y}_{2}\right) \neq \emptyset$ and fix $I \in \mathcal{I}\left(\bar{x}_{1}, \bar{x}_{2}, \bar{y}_{1}, \bar{y}_{2}\right)$.

For $z=x, y$ we have: $I / z_{2} \in{\overline{k\left(z_{1} / z_{2}\right)^{F}}}^{\times}$, so that there exists $A_{z} \in{\overline{k\left(z_{1} / z_{2}\right)^{F}}}^{\times}$with

$$
\frac{d\left(I / z_{2}\right)}{I / z_{2}}=A_{z} \frac{d\left(z_{1} / z_{2}\right)}{z_{1} / z_{2}}
$$

(here, we use $\left.z_{1} / z_{2} \notin F^{p}\right)$. Equivalently,

$$
\frac{d I}{I}=\frac{d z_{2}}{z_{2}}\left(1-A_{z}\right)+\frac{d z_{1}}{z_{1}} A_{z}
$$

We deduce

$$
A_{x} \frac{d x_{1}}{x_{1}}-A_{y} \frac{d y_{1}}{y_{1}}=\left(A_{x}-1\right) \frac{d x_{2}}{x_{2}}-\left(A_{y}-1\right) \frac{d y_{2}}{y_{2}} \in F d x_{1} \cap F d x_{2}=0 .
$$

Whence, setting $d y_{i}=y_{i}^{\prime} d x_{i}$ with $y_{i}^{\prime} \in \overline{k\left(x_{i}\right)^{F}}$ (here, we use $x_{i} \notin F^{p}$ ) and using that $d x_{1}, d x_{2}$ are linearly independent over $F$, we obtain

$$
A_{x}=A_{y} \frac{x_{1} y_{1}^{\prime}}{y_{1}}=\left(A_{y}-1\right) \frac{x_{2} y_{2}^{\prime}}{y_{2}}+1
$$

Set

$$
f_{i}:=\frac{x_{i} y_{i}^{\prime}}{y_{i}} \in{\overline{k\left(x_{i}\right)^{F}}}^{\times}, i=1,2 .
$$

We obtain

$$
A_{x}=A_{y} f_{1}, \quad A_{y}\left(f_{1}-f_{2}\right)=1-f_{2}
$$


By Lemma 25 (i) below and the fact that $y_{2} \varkappa_{p} x_{2}, 1-f_{2} \neq 0$ hence $f_{1}-f_{2}, A_{y} \neq 0$. As a result the second equation in (3.3) can be rewritten

$$
A_{y}=\frac{1-f_{2}}{f_{1}-f_{2}}
$$

So, setting $d f_{i}=f_{i}^{\prime} d x_{i}$ with $f_{i}^{\prime} \in \overline{k\left(x_{i}\right)^{F}}, i=1,2$ we get

$$
\frac{d A_{y}}{A_{y}}=\frac{f_{1}^{\prime}}{f_{2}-f_{1}} d x_{1}+\frac{\left(1-f_{1}\right) f_{2}^{\prime}}{\left(1-f_{2}\right)\left(f_{1}-f_{2}\right)} d x_{2} .
$$

We also have $A_{y} \in \overline{k\left(y_{1} / y_{2}\right)^{F}} \times$ so that there exists $\alpha \in \overline{k\left(y_{1} / y_{2}\right)^{F}}$ with

$$
\frac{d A_{y}}{A_{y}}=\alpha\left(\frac{y_{1}^{\prime}}{y_{1}} d x_{1}-\frac{y_{2}^{\prime}}{y_{2}} d x_{2}\right) .
$$

(here we use $y_{1} / y_{2} \notin F^{p}$ ). Since $d x_{1}, d x_{2}$ are linearly independent over $F$, one gets

$$
\alpha=\frac{y_{1} f_{1}^{\prime}}{y_{1}^{\prime}\left(f_{2}-f_{1}\right)}=\frac{y_{2}\left(1-f_{1}\right) f_{2}^{\prime}}{y_{2}^{\prime}\left(1-f_{2}\right)\left(f_{2}-f_{1}\right)}
$$

whence

$$
c:=\frac{y_{1} f_{1}^{\prime}}{y_{1}^{\prime}\left(1-f_{1}\right)}=\frac{y_{2} f_{2}^{\prime}}{y_{2}^{\prime}\left(1-f_{2}\right)} \in \overline{k\left(x_{1}\right)^{F}} \cap \overline{k\left(x_{2}\right)^{F}}=k .
$$

By Lemma 25 (ii) below and the fact that $y_{1} \varkappa_{p} x_{1}$, we have $f_{1} \notin F^{p}$ hence $c \neq 0$. Recalling that $f_{i}:=\frac{x_{i} y_{i}^{\prime}}{y_{i}}, i=1,2$ we eventually get

$$
x_{i} f_{i}^{\prime}=c f_{i}\left(1-f_{i}\right) .
$$

Now, the last steps of the proof are as follows.

- Step 1. Considering (3.4) for $i=1$ and using that $x_{1} \in F^{\times}$is $F / k$-regular, we show that the parameter $c$ necessarily lies in $\mathbb{Z}$ and can be taken satisfying (1.1); we denote it $c:=m$. Once this is settled, one can easily solve (3.4) and determine $y_{i}^{m}, i=1,2$.

- Step 2. Using the relations between $I, A_{x}, A_{y}, f_{1}, f_{2}$, we show that

$$
I^{m}=\alpha^{p}\left(x_{1}^{m}-\frac{\beta_{1}^{p}}{\beta_{2}^{p}} x_{2}^{m}\right)
$$

for some $\alpha \in \overline{k\left(\frac{x_{1}}{x_{2}}\right)^{F}}, \beta_{i} \in \overline{k\left(x_{i}\right)^{F}}, i=1,2$.

- Step 3. Using that $I / x_{2} \in \overline{k\left(\frac{x_{1}}{x_{2}}\right)^{F}}$, we show that there exists $N \in \mathbb{Z}$ such that $\beta_{i}=c_{i} x_{i}^{N}$ for some $c_{i} \in k^{\times}, i=1,2$.

\subsubsection{Step 1. Write}

$$
f_{1}=\frac{a_{1} A_{1}^{p}}{b_{1} B_{1}^{p}}
$$

with $A_{1}, B_{1}, a_{1}, b_{1} \in k\left[x_{1}\right], a_{1} A_{1}, b_{1} B_{1} \in k\left[x_{1}\right]$ coprime, $a_{1}, b_{1}, B_{1} \in k\left[x_{1}\right]$ monic and $a_{1}, b_{1} \in k\left[x_{1}\right]$ with zeros of multiplicities at most $p-1$. Then $x_{1} f_{1}^{\prime}=c f_{1}\left(1-f_{1}\right)$ can be rewritten as

$$
x_{1}\left(a_{1}^{\prime} b_{1}-a_{1} b_{1}^{\prime}\right) B_{1}^{p}=c a_{1}\left(B_{1}^{p} b_{1}-A_{1}^{p} a_{1}\right) .
$$

By considering the multiplicity of a non-zero root of $a_{1}$, we see that $a_{1}=x_{1}^{m}$ for some $0 \neq m \in \mathbb{Z}$ with $\frac{1-p}{2} \leq m \leq \frac{p-1}{2}$ so that, for $b_{1}$, we obtain

$$
\left((m-c) b_{1}-x_{1} b_{1}^{\prime}\right) B_{1}^{p}=-c x_{1}^{m} A_{1}^{p} .
$$


Since $B_{1}^{p}$ and $a_{1} A_{1}^{p}=x_{1}^{m} A_{1}^{p}$ are corpime and $B_{1}$ is monic, $B_{1}=1$ and (3.5) becomes

$$
(m-c) b_{1}-x_{1} b_{1}^{\prime}=-c x_{1}^{m} A_{1}^{p} .
$$

- If $m \neq 0$, evaluating at 0 and using that $b_{1}(0) \neq 0$ (since $a_{1}=x_{1}^{m}$ and $b_{1}$ are coprime), one gets $c=m$;

- If $m=0$, differentiating (3.6), one gets

$$
(c+1) b_{1}^{\prime}-+x_{1} b_{1}^{\prime \prime}=0
$$

and writing $b_{1}=\sum_{j} b_{1, j} x_{1}^{j}$ this yields

$$
\sum_{j} j(c+j) b_{1, j} x_{1}^{j-1}=0 .
$$

Again, since $b_{1} \notin k\left[x_{1}^{p}\right]$ by assumption, there exists $p \nmid\left\lceil j\right.$ such that $b_{1, j} \neq 0$, which forces $c=-j$.

In any case, one may now write $c=m$ for some $0 \neq m \in \mathbb{Z}$ with $\frac{1-p}{2} \leq m \leq \frac{p-1}{2}$ hence for $i=1,2$, (3.4) can be rewritten

$$
\left(\frac{\left(1-f_{i}\right) x_{i}^{m}}{f_{i}}\right)^{\prime}=0
$$

hence

$$
\frac{\left(1-f_{i}\right) x_{i}^{m}}{f_{i}}=\beta_{i}^{p}
$$

for some $\beta_{i} \in \overline{k\left(x_{i}\right)^{F}}$ or, equivalently,

$$
\frac{x_{i} y_{i}^{\prime}}{y_{i}}=f_{i}=\frac{x_{i}^{m}}{x_{i}^{m}+\beta_{i}^{p}}
$$

Whence

$$
\frac{\left(x_{i}^{m}+\beta_{i}^{p}\right)^{\prime}}{x_{i}^{m}+\beta_{i}^{p}}=m \frac{x_{i}^{m-1}}{x_{i}^{m}+\beta_{i}^{p}}=\frac{\left(y_{i}^{m}\right)^{\prime}}{y_{i}^{m}}
$$

and

$$
d\left(\frac{y_{i}^{m}}{\left(x_{i}^{m}+\beta_{i}^{p}\right)}\right)=0
$$

that is

$$
y_{i}^{m}=\alpha_{i}^{p}\left(x_{i}^{m}+\beta_{i}^{p}\right)
$$

for some $\alpha_{i} \in \overline{k\left(x_{i}\right)^{F}}$.

Remark 23. If $p=0$, we obtain that there exists $0 \neq m \in \mathbb{Z}$ such that $y_{1}^{m}=\alpha_{1}\left(x_{1}^{m}+\beta_{1}\right)$ for some $\alpha_{1}, \beta_{1} \in k^{\times}$. Then the factoriality of $k\left[x_{1}\right]$ and the fact that $x_{1} \chi_{0} y_{1}$ yields $m= \pm 1$. If $p>0$, we are not able to ensure $m= \pm 1$. 
3.4.2. Step 2. Then,

$$
A_{y}=\frac{1-f_{2}}{f_{1}-f_{2}}=\frac{\beta_{2}^{p}\left(x_{1}^{m}+\beta_{1}^{p}\right)}{\beta_{2}^{p} x_{1}^{m}-\beta_{1}^{p} x_{2}^{m}}
$$

and

$$
A_{x}=A_{y} f_{1}=\left(1-\frac{\beta_{1}^{p}}{\beta_{2}^{p}}\left(\frac{x_{1}}{x_{2}}\right)^{-m}\right)^{-1} .
$$

So, writing $\beta:=\frac{\beta_{1}}{\beta_{2}}, x:=\frac{x_{1}}{x_{2}}$ and $J=J(x):=\frac{I}{x_{2}}$ one gets

$$
\frac{\left(J^{m}\right)^{\prime}}{J^{m}}=\frac{m x^{m-1}}{x^{m}-\beta^{p}}=\frac{\left(x^{m}-\beta^{p}\right)^{\prime}}{x^{m}-\beta^{p}}
$$

Whence

$$
J^{m}=\alpha^{p}\left(x^{m}-\beta^{p}\right)
$$

for some $\alpha \in \overline{k\left(\frac{x_{1}}{x_{2}}\right)^{F}}$ and

$$
I^{m}=\alpha^{p}\left(x_{1}^{m}-\beta^{p} x_{2}^{m}\right)=\alpha^{p}\left(x_{1}^{m}-\frac{\beta_{1}^{p}}{\beta_{2}^{p}} x_{2}^{m}\right)
$$

with $\alpha \in \overline{k\left(\frac{x_{1}}{x_{2}}\right)^{F}}$ and $\beta_{i} \in \overline{k\left(x_{i}\right)^{F}}, i=1,2$.

3.4.3. Step 3. The assumption $I / x_{2} \in \overline{k\left(\frac{x_{1}}{x_{2}}\right)^{F}}$ forces $\beta=\frac{\beta_{1}}{\beta_{2}} \in{\overline{k\left(\frac{x_{1}}{x_{2}}\right)^{F}}}^{\times}$. This in turn imposes

Lemma 24. $\beta_{1} \in k^{\times} x_{1}^{N}, \beta_{2} \in k^{\times} x_{2}^{N}$ for some $N \in \mathbb{Z}$.

Proof. Again, write $x:=\frac{x_{1}}{x_{2}}$. Up to replacing $\beta$ with $\beta w^{N}$ for some $N \in \mathbb{Z}$, one may assume 0 is neither a zero nor a pole of $\beta$, as a function in $x$. We are going to show that, necessarily, $\beta_{1}, \beta_{2} \in k^{\times}$. Let

$$
P_{\epsilon}(x, T)=T^{d}+\sum_{0 \leq i \leq d-1} a_{\epsilon, i}(x) T^{i} \in k(x)[T]
$$

be the monic minimal polynomial of $\beta^{\epsilon}$ over $k(x)$ for $\epsilon= \pm 1$. If $\beta_{1} \notin k^{\times}$, then $\beta_{1}$ or $\beta_{1}^{-1}$ admits at least one zero $\lambda \neq 0$. So the relations

$$
\begin{gathered}
\beta_{2}^{-d}+\sum_{0 \leq i \leq d-1} a_{1, i}(x)\left(\beta_{1}^{-1}\right)^{d-i} \beta_{2}^{-i}=0 \\
\beta_{2}^{d}+\sum_{0 \leq i \leq d-1} a_{-1, i}(x) \beta_{1}^{d-i} \beta_{2}^{i}=0
\end{gathered}
$$

yield $\beta_{2}^{-d}=0$ or $\beta_{2}^{d}=0$ : a contradiction. This shows that $\beta_{1} \in k^{\times}$. Then, as $x_{2}$ and $w$ are algebraically independent, $\beta_{2} \in \overline{k(x)^{F}} \cap \overline{k\left(x_{2}\right)^{F}}=k$.

This concludes the proof of Lemma 22 .

Lemma 25. Let $x \in F^{\times} \backslash F^{\times p}$ and $y \in{\overline{k(x)^{F}}}^{\times}$. Write $d y=y^{\prime} d x$. Then

(i) $\frac{x y^{\prime}}{y}=m \Rightarrow y \in F^{\times p} x^{m}, m \in \mathbb{Z}$

(ii) $y \in k(x)^{\times}$and $\frac{x y^{\prime}}{y} \in F^{\times p} \Rightarrow y \in F^{\times p} x^{\mathbb{Z}}$ (in particular, $x \sim_{p} y$ ). 
Proof. For (i), just observe that $\frac{x y^{\prime}}{y}=m$ if and only if

$$
d\left(\frac{y}{x^{m}}\right)=\frac{x^{m} y^{\prime}-m x^{m-1} y}{x^{2 m}}=0
$$

For (ii), write $y=\frac{a}{b} A^{p}$ with $0 \neq a, b \in k[x]$ coprime and with zeros of multiplicities at most $p-1$ and $a$ monic (if $p=0$, just impose $a, b \in k[x]$ to be coprime and $a$ monic). By assumption there exist $u, v \in k[x]$ coprime such that

$$
x\left(a^{\prime} b-a b^{\prime}\right) v^{p}=u^{p} a b .
$$

Assume $a$ has a non-zero root $\alpha$ of multiplicity $1 \leq n_{\alpha} \leq p-1$. Then, since $a$ and $b$ are coprime, on the left hand side, the multiplicity of $\alpha$ is congruent to $n_{\alpha}-1(\bmod p)$, while, one the right hand side, the multiplicity of $\alpha$ is congruent to $n_{\alpha}(\bmod p)$ : a contradiction. This shows there exists $0 \leq m \leq p-1$ such that $a=x^{m}$. The equation thus becomes

$$
\left(m b-x b^{\prime}\right) v^{p}=u^{p} b
$$

or, equivalently,

$$
b\left(m^{\frac{1}{p}} v-u\right)^{p}=\left(m v^{p}-u^{p}\right) b=x b^{\prime} v^{p}
$$

(recall $k$ is perfect). Again, considering the multiplicity of a non-zero root of $b$, one sees that $b \in k^{\times} x^{n}$ for some integer $0 \leq n \leq p-1$. As a result $y=\frac{a}{b} A^{p} \in F^{\times p} x^{\mathbb{Z}}$ as claimed.

3.5. Recovering lines in $F^{\times} / p, F^{\prime \times} / p$ up to powers. Let $k, k^{\prime}$ be perfect fields of characteristic $p \geq 0$, let $F / k, F^{\prime} / k^{\prime}$ be finitely generated regular field extensions of transcendence degree $\geq 2$ and let

$$
\bar{\psi}: F^{\times} / k^{\times} \stackrel{\sim}{\rightarrow} F^{\prime \times} / k^{\prime \times}
$$

be a group isomorphism preserving algebraic dependence. Write again

$$
\bar{\psi}: F^{\times} / p \stackrel{\sim}{\rightarrow} F^{\prime \times} / p
$$

for the group isomorphism induced by $\bar{\psi}$.

Proposition 26. There exists $m \in \mathbb{Z}$ satisfying (1.1) such that for every $\bar{x}_{1} \neq \bar{x}_{2} \in F^{\times} / p$,

$$
\bar{\psi}\left(\mathfrak{l}_{F^{p}}\left(\bar{x}_{1}, \bar{x}_{2}\right)\right)^{m}=\mathfrak{l}_{F^{\prime} p}\left(\bar{\psi}\left(\bar{x}_{1}\right)^{m}, \bar{\psi}\left(\bar{x}_{2}\right)^{m}\right)
$$

Proof. For simplicity, write $\bar{x}^{\prime}:=\bar{\psi}(\bar{x})$. We proceed in two steps.

- Step 1: We first show there exists $m \in \mathbb{Z}$ satisfying (1.1) such that for every $\bar{x}_{1} \neq \bar{x}_{2} \in F^{\times} / p$,

$$
\bar{\psi}\left(\mathfrak{l}_{F^{p}}\left(\bar{x}_{1}, \bar{x}_{2}\right)\right)^{m} \subset \mathfrak{l}_{F^{\prime} p}\left(\bar{x}_{1}^{\prime m}, \bar{x}_{2}^{\prime m}\right)
$$

By Lemma 19, one may assume $\left(\bar{x}_{1}^{\prime}, \bar{x}_{2}^{\prime}\right) \in F^{\prime \times} / k^{\prime \times}$ is a good pair. Since

$$
\mathfrak{l}_{F^{p}}\left(\bar{x}_{1}, \bar{x}_{2}\right)=\bigcup_{\alpha \in F^{\times}} F^{p \times} \mathfrak{l}_{k}\left(\bar{x}_{1}, \bar{\alpha}^{p} \bar{x}_{2}\right) .
$$

and for every $\alpha \in F^{\times},\left(\bar{x}_{1}^{\prime}, \bar{\alpha}^{p} \bar{x}_{2}^{\prime}\right) \in F^{\prime \times} / k^{\prime \times}$ is again a good pair, it is enough to prove that there exists $m \in \mathbb{Z}$ satisfying (1.1) such that for every $\bar{x}_{1} \neq \bar{x}_{2} \in F^{\times} / p$ for which $\left(\bar{x}_{1}^{\prime}, \bar{x}_{2}^{\prime}\right) \in F^{\prime \times} / k^{\prime \times}$ is a good pair, one has

$$
\bar{\psi}\left(\mathfrak{l}_{k}\left(\bar{x}_{1}, \bar{x}_{2}\right)\right)^{m} \subset \mathfrak{l}_{F^{\prime} p}\left(\bar{x}_{1}^{\prime m}, \bar{x}_{2}^{\prime m}\right) .
$$

Write

$$
\mathfrak{I}\left(\bar{x}_{1}^{\prime}, \bar{x}_{2}^{\prime}\right)_{m}:=\left\{I^{\prime} \in \mathfrak{I}\left(\bar{x}_{1}^{\prime}, \bar{x}_{2}^{\prime}\right) \mid I^{\prime m} \in \mathfrak{l}_{F^{\prime} p}\left(\bar{x}_{1}^{\prime m}, \bar{x}_{2}^{\prime m}\right)\right\} .
$$


From Lemma 22 and Lemma 28 below, one has

$$
\Im\left(\bar{x}_{1}^{\prime}, \bar{x}_{2}^{\prime}\right)=\left\{\bar{x}_{1}^{\prime}, \bar{x}_{2}^{\prime}\right\} \bigsqcup_{m}\left(\Im\left(\bar{x}_{1}^{\prime}, \bar{x}_{2}^{\prime}\right)_{m} \backslash\left\{\bar{x}_{1}^{\prime}, \bar{x}_{2}^{\prime}\right\}\right),
$$

where the union is over all $m \in \mathbb{Z}$ satisfying (1.1) and for every $I^{\prime} \in \mathfrak{I}\left(\bar{x}_{1}^{\prime}, \bar{x}_{2}^{\prime}\right)_{m}$, one has

$$
\mathfrak{l}\left(\overline{I^{\prime}}, \bar{x}_{i}^{\prime}\right)^{m} \subset \mathfrak{l}_{F^{\prime} p}\left(1, \bar{x}_{i}^{\prime m}\right), i=1,2 .
$$

From Lemma 27 below, $\mathfrak{l}_{k}\left(\bar{x}_{1}, \bar{x}_{2}\right) \subset \mathfrak{I}\left(\bar{x}_{1}, \bar{x}_{2}\right)$ so that $\bar{\psi}\left(\mathfrak{l}_{k}\left(\bar{x}_{1}, \bar{x}_{2}\right)\right) \subset \mathfrak{I}\left(\bar{x}_{1}^{\prime}, \bar{x}_{2}^{\prime}\right)$. Fix $I \in \mathfrak{l}_{k}\left(\bar{x}_{1}, \bar{x}_{2}\right)$, $\bar{I} \neq \bar{x}_{1}, \bar{x}_{2}$ in $F^{\times} / p$ and let $m:=m\left(\bar{x}_{1}, \bar{x}_{2}, \bar{I}\right) \in \mathbb{Z}$ be the unique integer satisfying (1.1) such that $I^{\prime} \in \mathfrak{I}\left(\bar{x}_{1}^{\prime}, \bar{x}_{2}^{\prime}\right)_{m}$. For $i=1,2$, one has $\mathfrak{l}_{k}\left(1, x_{i}\right) \subset \mathfrak{l}\left(\bar{I}, \bar{x}_{i}\right)$ hence

$$
\bar{\psi}\left(\mathfrak{l}_{k}\left(1, x_{i}\right)\right)^{m} \subset \bar{\psi}\left(\mathfrak{l}\left(\bar{I}, \bar{x}_{i}\right)\right)^{m} \subset \mathfrak{l}\left(\bar{I}^{\prime}, \bar{x}_{i}^{\prime}\right)^{m} \subset \mathfrak{l}_{F^{\prime}}\left(1, \bar{x}_{i}^{\prime m}\right) .
$$

By Lemma 28, this characterizes $m$ as the unique integer satisfying (1.1) such that

$$
\bar{\psi}\left(\mathfrak{l}_{k}\left(1, x_{i}\right)\right)^{m} \subset \mathfrak{l}_{F^{\prime} p}\left(1, \bar{x}_{i}^{\prime m}\right) .
$$

Hence $m$ is uniquely determined by any of the two sets $\bar{\psi}\left(\mathfrak{l}_{k}\left(1, x_{i}\right)\right), i=1,2$ and, in particular, does not depend on $I$. As a result:

$$
\bar{\psi}\left(\mathfrak{l}_{k}\left(\bar{x}_{1}, \bar{x}_{2}\right)\right)^{m} \subset \mathfrak{l}_{F^{\prime}}\left(\bar{x}_{1}^{\prime m}, \bar{x}_{2}^{\prime m}\right) .
$$

In fact, $m$ does not depend on the line $\mathfrak{l}_{k}\left(\bar{x}_{1}, \bar{x}_{2}\right)$ either. Indeed, let $\mathfrak{l}_{k}\left(\bar{y}_{1}, \bar{y}_{2}\right)$ be any other line such that $\left(\bar{y}_{1}^{\prime}, \bar{y}_{2}^{\prime}\right) \in F^{\prime \times} / k^{\prime \times}$ is a good pair and let $n \in \mathbb{Z}$ satisfying (1.1) be the attached integer. Then, necessarily, at least one of the two pairs $\left(\bar{x}_{1}^{\prime}, \bar{y}_{1}^{\prime}\right),\left(\bar{x}_{1}^{\prime}, \bar{y}_{2}^{\prime}\right)$ - say $\left(\bar{x}_{1}^{\prime}, \bar{y}_{1}^{\prime}\right)$ - is a good pair; let $r \in \mathbb{Z}$ satisfying (1.1) be the attached integer. Then, by considering $\bar{\psi}\left(\mathfrak{l}_{k}\left(1, x_{1}\right)\right)$, one has $m=r$ and by considering $\bar{\psi}\left(\mathfrak{l}_{k}\left(1, y_{1}\right)\right)$, one has $n=r$.

- Step 2: Since the situation is symmetric in $F^{\times} / k^{\times}$and $F^{\prime \times} / k^{\prime \times}$ (here, we use that $\bar{\psi}$ preserves algebraic dependence if and only if $\bar{\psi}^{-1}$ does, by the very definition of 'preserving algebraic dependence'), there exists $m^{\prime} \in \mathbb{Z}$ satisfying conditions (1.1), such that for every $\bar{x}_{1}^{\prime}, \bar{x}_{2}^{\prime} \in F^{\prime \times} / k^{\prime \times}$ with $\bar{x}_{1}^{\prime} \neq \bar{x}_{2}^{\prime}$ in $F^{\prime \times} / p$ one also has

$$
\bar{\psi}^{-1}\left(\mathfrak{l}_{F^{\prime} p}\left(\bar{x}_{1}^{\prime}, \bar{x}_{2}^{\prime}\right)\right)^{m^{\prime}} \subset \mathfrak{l}_{F^{p}}\left(\bar{\psi}^{-1}\left(\bar{x}_{1}^{\prime}\right)^{m^{\prime}}, \bar{\psi}^{-1}\left(\bar{x}_{2}^{\prime}\right)^{m^{\prime}}\right)
$$

in $F^{\times} / p$. As a result

$$
\mathfrak{l}_{F^{p}}\left(\bar{x}_{1}, \bar{x}_{2}\right)^{m m^{\prime}}=\bar{\psi}^{-1}\left(\bar{\psi}\left(\mathfrak{l}_{F^{p}}\left(\bar{x}_{1}, \bar{x}_{2}\right)\right)^{m}\right)^{m^{\prime}} \subset \bar{\psi}^{-1}\left(\mathfrak{l}_{F^{\prime}}\left(\bar{\psi}\left(\bar{x}_{1}\right)^{m}, \bar{\psi}\left(\bar{x}_{2}\right)^{m}\right)\right)^{m^{\prime}} \subset \mathfrak{l}_{F}\left(\bar{x}_{1}^{m m^{\prime}}, \bar{x}_{2}^{m m^{\prime}}\right)
$$

In particular, if $\bar{x}_{1}, \bar{x}_{2} \in F^{\times} / k^{\times}$are such that $d x_{1}, d x_{2}$ are linearly independent over $F$, we obtain

$$
\left(x_{1}+x_{2}\right)^{m m^{\prime}}=a_{1}^{p} x_{1}^{m m^{\prime}}+a_{2}^{p} x_{2}^{m m^{\prime}} \text { for some } a_{1}, a_{2} \in F^{\times}
$$

hence

$$
m m^{\prime}\left(x_{1}+x_{2}\right)^{m m^{\prime}-1}\left(d x_{1}+d x_{2}\right)=m m^{\prime}\left(a_{1}^{p} x_{1}^{m m^{\prime}-1} d x_{1}+a_{2}^{p} x_{2}^{m m^{\prime}-1} d x_{2}\right)
$$

and

This forces

$$
\left(x_{1}+x_{2}\right)^{m m^{\prime}-1}=a_{1}^{p} x_{1}^{m m^{\prime}-1}=a_{2}^{p} x_{2}^{m m^{\prime}-1} .
$$

$$
m m^{\prime} \equiv 1(\bmod p)
$$

Now, let $\bar{x}_{1}, \bar{x}_{2} \in F^{\times} / k^{\times}$with $\bar{x}_{1} \neq \bar{x}_{2}$ in $F^{\times} / p$ and apply (3.8) to $\bar{x}_{i}^{\prime}=\bar{\psi}_{1}\left(\bar{x}_{i}\right)^{m}, i=1,2$. Using $m m^{\prime} \equiv 1(\bmod p)$, we obtain

$$
\bar{\psi}^{-1}\left(\mathfrak{l}_{F^{\prime} p}\left(\bar{\psi}\left(\bar{x}_{1}\right)^{m}, \bar{\psi}\left(\bar{x}_{2}\right)^{m}\right)\right) \subset \mathfrak{l}_{F^{p}}\left(\bar{x}_{1}, \bar{x}_{2}\right)^{m}
$$


in $F^{\times} / p$. This concludes the proof of Proposition 26 .

Lemma 27. For every $\bar{x}_{1}, \bar{x}_{2} \in F^{\times} / k^{\times}$such that $\bar{x}_{1} \neq \bar{x}_{2} \in F^{\times} / p$, one has $\mathfrak{l}_{k}\left(\bar{x}_{1}, \bar{x}_{2}\right) \subset \mathfrak{I}\left(\bar{x}_{1}, \bar{x}_{2}\right)$. Proof. Fix $c \in k$. On the one hand $x_{1}-c x_{2}=\left(\frac{x_{1}}{x_{2}}-c\right) x_{2} \in k\left(x_{1} / x_{2}\right)^{\times} x_{2}$ and on the other hand, for every $c_{1}, c_{2} \in k^{\times}$such that $c=c_{1} / c_{2}$,

$$
x_{1}-c x_{2}=\left(\frac{x_{1}-c_{1}}{x_{2}-c_{2}}-c\right)\left(x_{2}-c_{2}\right) \in k\left(\frac{x_{1}-c_{1}}{x_{2}-c_{2}}\right)^{\times}\left(x_{2}-c_{2}\right) .
$$

To ensure that $x_{1}-c x_{2} \in \mathfrak{I}\left(\bar{x}_{1}, \bar{x}_{2}\right)$ we have to check that $x_{i} \varkappa_{p} x_{i}-c_{i}$ for $i=1,2$. Otherwise, there would be nonzero integers $a_{i}, b_{i} \in \mathbb{Z}$ and $\alpha_{i} \in \overline{k\left(x_{i}\right)^{F}}$ such that

$$
\frac{\left(x_{i}-c_{i}\right)^{b_{i}-1} x_{i}^{a_{i}-1}\left(b_{i} x_{i}-a_{i}\left(x_{i}-c_{i}\right)\right)}{x_{i}^{2 a_{i}}}=d\left(\frac{\left(x_{i}-c_{i}\right)^{b_{i}}}{x_{i}^{a_{i}}}\right)=0,
$$

which forces $a_{i}=b_{i}=0$ : a contradiction.

Lemma 28. For every $\bar{x} \in F^{\times} / k^{\times}$such that $\bar{x} \neq 1$ in $F^{\times} / p$ and for $m \neq n \in \mathbb{Z}$ satisfying (1.1),

$$
\mathfrak{l}_{F^{p}}\left(1, \bar{x}^{m}\right)^{n} \cap \mathfrak{l}_{F^{p}}\left(1, \bar{x}^{n}\right)^{m} \subset\{1\} \cup F^{p \times} x^{m n} .
$$

Proof. If $\mathfrak{l}_{F^{p}}\left(1, \bar{x}^{m}\right)^{n} \cap \mathfrak{l}_{F^{p}}\left(1, \bar{x}^{n}\right)^{m} \backslash\{1\} \neq \emptyset$, there exist $a, b, c, d \in F, b \neq 0 \neq d$, such that

$$
\left(a^{p}+b^{p} x^{m}\right)^{n}=\left(c^{p}+d^{p} x^{n}\right)^{m} .
$$

Taking the logarithmic differentials and using that $d x \neq 0$, one gets

$$
\frac{b^{p} x^{m-1}}{a^{p}+b^{p} x^{m}}=\frac{d^{p} x^{n-1}}{c^{p}+d^{p} x^{n}}
$$

hence $c^{p} b^{p} x^{m-1}=a^{p} d^{p} x^{n-1}$, which is only possible if $m=n$ or $c b=a d=0$. But, in turn, $c b=a d=0$ is possible only if $a=c=0$.

\subsection{End of the proof of Theorem 4 .}

From Proposition 26 and Lemma 29 (ii), applied to the field extensions $F / F^{p}$ and $F^{\prime} / F^{\prime p}$, there exist an integer $m \in \mathbb{Z}$ satisfying conditions (1.1), and a unique field isomorphism $\phi: F \stackrel{\sim}{\rightarrow} F^{\prime}$ such that the following diagram

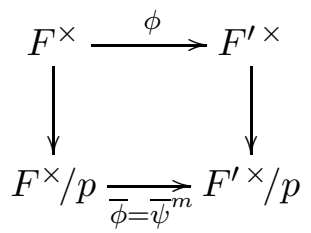

commutes. This concludes the proof for $p=0$. For $p>0$, one needs to work more, using Lemma 24 .

Consider the group morphism

$$
\begin{array}{ll}
\bar{\epsilon}: F^{\times} / k^{\times} & \rightarrow F^{\prime \times p} / k^{\prime \times} \\
\bar{x} & \rightarrow \bar{\psi}(\bar{x})^{m} \bar{\phi}(\bar{x})^{-1} .
\end{array}
$$

We are to show that $m= \pm 1$ and $\bar{\epsilon}$ is trivial.

Fix a set-theoretic lift $\psi: F^{\times} \rightarrow F^{\prime \times}$ of $\bar{\psi}: F^{\times} / k^{\times} \rightarrow F^{\prime \times} / k^{\prime \times}$ and set

$$
\begin{aligned}
\epsilon: F^{\times} & \rightarrow F^{\prime \times} \\
x & \rightarrow \psi(x)^{m} \phi(x)^{-1}
\end{aligned}
$$


Let $x_{1}, x_{2} \in F^{\times}$be such that $\left(\bar{x}_{1}^{\prime}, \bar{x}_{2}^{\prime}\right) \in F^{\prime \times} / k^{\prime \times}$ is a good pair. From Lemma 24 , one has

$$
\overline{\psi\left(x_{1}+x_{2}\right)^{m}}=\overline{\alpha^{p}\left(\psi\left(x_{1}\right)^{m}+\lambda\left(\frac{\psi\left(x_{1}\right)^{N p}}{\psi\left(x_{2}\right)^{N p}} \psi\left(x_{2}\right)^{m}\right)\right.}
$$

in $F^{\prime \times} / k^{\prime \times}$ for some $\alpha \in F^{\prime \times}, \lambda \in k^{\prime \times}$ and $N \in \mathbb{Z}$ (depending on $x_{1}, x_{2}$ ). Using the definition of $\epsilon$ and using that $\phi: F \rightarrow F^{\prime}$ is a field homomorphism (hence is compatible with the additive structure), one has

$$
\overline{\psi\left(x_{1}+x_{2}\right)^{m}}=\overline{\epsilon\left(x_{1}+x_{2}\right) \phi\left(x_{1}+x_{2}\right)}=\overline{\frac{\epsilon\left(x_{1}+x_{2}\right)}{\epsilon\left(x_{1}\right)} \psi\left(x_{1}\right)^{m}+\frac{\epsilon\left(x_{1}+x_{2}\right)}{\epsilon\left(x_{2}\right)} \psi\left(x_{2}\right)^{m}} .
$$

in $F^{\prime \times} / k^{\prime \times}$. This shows that

$$
\mu \alpha^{p}\left(\psi\left(x_{1}\right)^{m}+\lambda\left(\frac{\psi\left(x_{1}\right)^{N p}}{\psi\left(x_{2}\right)^{N p}} \psi\left(x_{2}\right)^{m}\right)=\frac{\epsilon\left(x_{1}+x_{2}\right)}{\epsilon\left(x_{1}\right)} \psi\left(x_{1}\right)^{m}+\frac{\epsilon\left(x_{1}+x_{2}\right)}{\epsilon\left(x_{2}\right)} \psi\left(x_{2}\right)^{m}\right.
$$

for some $\mu \in k^{\prime \times}$.

Since $\psi\left(x_{1}\right), \psi\left(x_{2}\right)$ are algebraically independent over $F^{\prime p}$ by assumption, and since $p \nmid m$, $\psi\left(x_{1}\right)^{m}, \psi\left(x_{2}\right)^{m}$ are linearly independent over $F^{\prime p}$ so that

$$
\overline{\frac{\epsilon\left(x_{1}+x_{2}\right)}{\epsilon\left(x_{1}\right)}}=\overline{\alpha^{p}}, \overline{\frac{\epsilon\left(x_{1}+x_{2}\right)}{\epsilon\left(x_{2}\right)}}=\overline{\alpha^{p} \frac{\psi\left(x_{1}\right)^{N p}}{\psi\left(x_{2}\right)^{N p}}} .
$$

Combining both equalities one obtains

$$
\bar{\epsilon}\left(\bar{x}_{1}\right)=\frac{\bar{\psi}\left(\bar{x}_{1}\right)^{N p}}{\bar{\psi}\left(\bar{x}_{2}\right)^{N p}} \bar{\epsilon}\left(\bar{x}_{2}\right) .
$$

Now fix two primes $p^{\prime} \neq p^{\prime \prime}$ distinct from $p$ and such that $p^{\prime} \not \equiv 1\left(\bmod p^{\prime \prime}\right)$, and apply the above to $x_{1}, x_{2}^{p^{\prime}}, x_{1}, x_{2}^{p^{\prime \prime}}$ to get

$$
\begin{aligned}
\bar{\epsilon}\left(\bar{x}_{1}\right) & =\frac{\bar{\psi}\left(\bar{x}_{1}\right)^{N^{\prime} p}}{\bar{\psi}\left(\bar{x}_{2}\right)^{N^{\prime} p^{\prime} p}} \bar{\epsilon}\left(\bar{x}_{2}\right)^{p^{\prime}} \\
\bar{\epsilon}\left(\bar{x}_{1}\right) & =\frac{\bar{\psi}\left(\bar{x}_{1}\right)^{N^{\prime \prime} p}}{\bar{\psi}\left(\bar{x}_{2}\right)^{N^{\prime \prime} p^{\prime \prime} p}} \bar{\epsilon}\left(\bar{x}_{2}\right)^{p^{\prime \prime}}
\end{aligned}
$$

for some $N^{\prime}, N^{\prime \prime} \in \mathbb{Z}$. Since the map $x^{\prime} \rightarrow x^{\prime p}$ is injective on $F^{\prime \times} / k^{\prime \times}$, we deduce

$$
\left(\frac{\bar{\psi}\left(\bar{x}_{1}\right)^{N-N^{\prime}}}{\bar{\psi}\left(\bar{x}_{2}\right)^{N-N^{\prime} p^{\prime}}}\right)^{p^{\prime \prime}-1}=\left(\frac{\bar{\psi}\left(\bar{x}_{1}\right)^{N-N^{\prime \prime}}}{\bar{\psi}\left(\bar{x}_{2}\right)^{N-N^{\prime \prime} p^{\prime \prime}}}\right)^{p^{\prime}-1} .
$$

Since $\bar{\psi}\left(\bar{x}_{1}\right), \bar{\psi}\left(\bar{x}_{2}\right)$ are multiplicatively independent, this forces

$$
\begin{aligned}
& \left(p^{\prime \prime}-p^{\prime}\right) N-\left(p^{\prime \prime}-1\right) N^{\prime}+\left(p^{\prime}-1\right) N^{\prime \prime}=0 \\
& \left(p^{\prime \prime}-p^{\prime}\right) N-p^{\prime}\left(p^{\prime \prime}-1\right) N^{\prime}+p^{\prime \prime}\left(p^{\prime}-1\right) N^{\prime \prime}=0
\end{aligned}
$$

Reducing modulo $p^{\prime \prime}$ and using that $p^{\prime} \not \equiv 1\left(\bmod p^{\prime \prime}\right)$, one sees that the matrix

$$
\left(\begin{array}{ccc}
\left(p^{\prime \prime}-p^{\prime}\right) & -\left(p^{\prime \prime}-1\right) & \left(p^{\prime}-1\right) \\
\left(p^{\prime \prime}-p^{\prime}\right) & p^{\prime}\left(p^{\prime \prime}-1\right) & p^{\prime \prime}\left(p^{\prime}-1\right)
\end{array}\right)
$$

has rank 2 . Since $(1,1,1)$ is a solution of the system above, we deduce $N=N^{\prime}=N^{\prime \prime}$. This implies

$$
\bar{\epsilon}\left(\overline{x_{2}}\right)^{\left(p^{\prime}-1\right)}=\bar{\psi}\left(\overline{x_{2}}\right)^{N p\left(p^{\prime}-1\right)} \text {, hence } \bar{\epsilon}\left(\bar{x}_{2}\right)=\bar{\psi}\left(\overline{x_{2}}\right)^{N p},
$$

and

$$
\bar{\epsilon}\left(\bar{x}_{1}\right)=\bar{\psi}\left(\bar{x}_{1}\right)^{N p} .
$$


In particular, this shows that $N$ does not depend on $x_{2}$. But it does not depend on $x_{1}$ either: if $y_{1} \in F^{\times}$is another element such that $\psi\left(y_{1}\right)$ is $F^{\prime} / k^{\prime}$-regular then either $d \psi\left(x_{1}\right), d \psi\left(y_{1}\right)$ are linearly independent over $F^{\prime}$ and one applies the above with $\left(x_{1}, x_{2}\right):=\left(x_{1}, y_{1}\right)$ or one can always find $x_{2} \in F^{\times}$such that $d \psi\left(x_{1}\right), d \psi\left(x_{2}\right)$ and $d \psi\left(y_{1}\right), d \psi\left(x_{2}\right)$ are linearly independent over $F^{\prime}$ and one applies the above with $\left(x_{1}, x_{2}\right):=\left(x_{1}, x_{2}\right)$ and $\left(x_{1}, x_{2}\right):=\left(y_{1}, x_{2}\right)$ respectively. Since, by Lemma 19 , for every $x \in F^{\times} \backslash F^{\times p}$ there exists $y \in F^{\times}$such that $(\bar{x}, \bar{y}) \in F^{\times} / k^{\times}$is a good pair, we deduce

$$
\bar{\epsilon}(\bar{x})=\bar{\psi}(\bar{x})^{N p}, x \in F^{\times} \backslash F^{\times p} .
$$

But by multiplicativity of $\bar{\epsilon}, \bar{\psi}$ this also holds for $x \in F^{\times p} \backslash k^{\times}$since such an $x$ can be written as $x=x_{0}^{p^{s}}$ for some $x_{0} \in F^{\times} \backslash F^{\times p}$ and integer $s \geq 1$. By definition of $\bar{\epsilon}$, this means

$$
\bar{\psi}(\bar{x})^{m-N p}=\bar{\phi}(\bar{x}), \bar{x} \in F^{\times} / k^{\times} .
$$

Since $\phi: F \stackrel{\sim}{\rightarrow} F^{\prime}$ is a field isomorphism, this is only possible if $m-N p= \pm 1$ (otherwise, the resulting morphism of groups $\bar{\phi}: F^{\times} / k^{\times} \rightarrow F^{\prime \times} / k^{\prime \times}$ would not be surjective). Since $m$ satisfies conditions (1.1) this forces $N=0, m= \pm 1$, hence

$$
\bar{\psi}(\bar{x})=\bar{\phi}(\bar{x})^{ \pm 1}, x \in F^{\times}
$$

as claimed.

\section{The Fundamental theorem of PROJECtive GeOMETRY}

Lemma 29. Let $k, k^{\prime}$ be fields and let $F / k$ and $F^{\prime} / k^{\prime}$ be field extensions. Let

$$
\phi: F^{\times} / k^{\times} \rightarrow F^{\prime \times} / k^{\prime \times}
$$

be a group morphism. Assume that:

(i) $\phi$ is injective and preserves collinearity: for any line $L \subset F^{\times} / k^{\times}$there is a line $L^{\prime} \subset$ $F^{\prime \times} / k^{\prime \times}$, such that $\phi(L) \subset L^{\prime}$, or that

(ii) $\phi$ is an isomorphism and preserves lines: for any line $L \subset F^{\times} / k^{\times}$there is a line $L^{\prime} \subset$ $F^{\prime \times} / k^{\prime \times}$, such that $\phi(L)=L^{\prime}$.

Assume that the image of $\phi$ is contained in no dimension $\leq 2$ projective subspace of $F^{\prime \times} / k^{\prime \times}$. Then, in case (i) (resp., in case (ii)), there is a unique field morphism (resp. isomorphism) $\Phi: F \rightarrow F^{\prime}$ such that the induced group morphism $\bar{\Phi}: F^{\times} / k^{\times} \rightarrow F^{\prime \times} / k^{\prime \times}$ coincides with $\phi$.

Lemma 29 is elementary and well-known to experts. See for instance [1, Chap. II, Thm. 2.26] for a classical formulation in the setting of finite dimensional vector spaces. For the convenience of the reader, we include here a proof in the setting of (not necessarily finite) field extensions.

Recall that for $x \in F^{\times}$we denote by $\bar{x}$ its image $\bar{x} \in F^{\times} / k^{\times}$.

\subsection{Definition of $\Phi$.}

4.1.1. Definition on $\{0,1\}$. Set $\Phi(0)=0, \Phi(1)=1$.

4.1.2. Definition on $F \backslash k$. For every $x \in F^{\times} \backslash k^{\times}$, we have

$$
\bar{\phi}(\overline{1+x}) \in \mathfrak{l}_{k^{\prime}}(1, \bar{\phi}(\bar{x})) \text {, }
$$

so that there exists a unique $\Phi(x) \in F^{\prime \times}$ such that

$$
\overline{\Phi(x)}=\bar{\phi}(\bar{x}) \text { and } \bar{\phi}(\overline{1+x})=\overline{1+\Phi(x)} \text {. }
$$


4.1.3. Definition on $k \backslash\{0,1\}$. For every $x \in F^{\times}$, set $\Phi_{x}(0)=0$. For every $\alpha \in k^{\times}$,

$$
\overline{\Phi(\alpha x)}=\bar{\phi}(\bar{x})=\overline{\Phi(x)}
$$

so that there exists a unique $\Phi_{x}(\alpha) \in k^{\prime \times}$ such that

$$
\Phi(\alpha x)=\Phi_{x}(\alpha) \Phi(x)
$$

Note that by definition $\Phi_{x}(1)=1, \Phi_{x}\left(k^{\times}\right) \subset k^{\prime \times}$.

Lemma 30. For every $x, y \in F^{\times} \backslash k^{\times}, \Phi_{x}=\Phi_{y}$.

Proof. $\quad \underline{\text { Case 1: }}$ The elements 1, $\Phi(x), \Phi(y)$ are linearly independent over $k^{\prime}$. In particular, for every $\alpha \in k^{\times}$,

$$
\mathfrak{l}_{k^{\prime}}(\overline{\Phi(x)}, \overline{\Phi(y)}) \neq \mathfrak{l}_{k^{\prime}}\left(\overline{1+\Phi_{x}(\alpha) \Phi(x)}, \overline{1+\Phi_{y}(\alpha) \Phi(y)}\right) .
$$

Since $\bar{\phi}$ preserves collinearity and

$$
\overline{(1+\alpha x)-(1+\alpha y)}=\overline{x-y} \in \mathfrak{l}_{k}(\bar{x}, \bar{y}) \cap \mathfrak{l}_{k}(\overline{1+\alpha x}, \overline{1+\alpha y})
$$

we see that

$$
\{\bar{\phi}(\overline{x-y})\}=\mathfrak{l}_{k^{\prime}}(\overline{\Phi(x)}, \overline{\Phi(y)}) \cap \mathfrak{l}_{k^{\prime}}\left(\overline{1+\Phi_{x}(\alpha) \Phi(x)}, \overline{1+\Phi_{y}(\alpha) \Phi(y)}\right)
$$

is independent of $\alpha$ while, on the other hand, a direct computation shows that for every $\alpha \in k^{\times}$,

$$
\left\{\overline{\Phi_{x}(\alpha) \Phi(x)-\Phi_{y}(\alpha) \Phi(y)}\right\}=\mathfrak{l}_{k^{\prime}}(\overline{\Phi(x)}, \overline{\Phi(y)}) \cap \mathfrak{l}_{k^{\prime}}\left(\overline{1+\Phi_{x}(\alpha) \Phi(x)}, \overline{1+\Phi_{y}(\alpha) \Phi(y)}\right) .
$$

This forces $\Phi_{x}(\alpha)=\Phi_{y}(\alpha)$.

Case 2:The elements $1, \Phi(x), \Phi(y)$ are linearly dependent over $k^{\prime}$. Then, by assumption, there exists $z \in F^{\times}$such that $1, \Phi(x), \Phi(z)$ (equivalently $1, \Phi(y), \Phi(z)$ ) are linearly independent over $k^{\prime}$. By the above $\Phi_{x}=\Phi_{z}$ and $\Phi_{y}=\Phi_{z}$.

In particular, for every $\alpha, \beta \in k^{\times}, x \in F^{\times}$, we have

$$
\Phi(\alpha \beta) \Phi(x)=\Phi(\alpha \beta x)=\Phi(\alpha) \Phi(\beta x)=\Phi(\alpha) \Phi(\beta) \Phi(x)
$$

whence

$$
\Phi(\alpha \beta)=\Phi(\alpha) \Phi(\beta) .
$$

Since by definition $\Phi(1)=1$, this hows that $\Phi: k^{\times} \rightarrow k^{\prime \times}$ is a group morphism.

4.2. We have to show that the map $\Phi: F \rightarrow F^{\prime}$ defined in Subsection 4.1 is a field morphism. By definition, we already have $\Phi(0)=0, \Phi(1)=1$ and for $\alpha \in k, x \in F^{\times} \backslash k^{\times}$,

$$
\Phi(\alpha x)=\Phi(\alpha) \Phi(x), \overline{\Phi(x)}=\bar{\phi}(\bar{x}), \overline{\Phi(1+x)}=\bar{\phi}(\overline{1+x})=\overline{1+\Phi(x)} .
$$

Lemma 31. For every $x, y \in F$ we have

$$
\Phi(x+y)=\Phi(x)+\Phi(y) .
$$


Proof. We may assume $x, y \neq 0$.

Case 1: $1, \Phi(x), \Phi(y)$ are linearly independent over $k^{\prime}$. Then

$$
\mathfrak{l}_{k^{\prime}}(\overline{1+\Phi(x)}, \overline{\Phi(y)}) \neq \mathfrak{l}_{k^{\prime}}(\overline{\Phi(x)}, \overline{1+\Phi(y)}) .
$$

Since $\bar{\phi}$ preserves collinearity and

$$
\overline{1+x+y} \in \mathfrak{l}_{k}(\overline{1+x}, \bar{y}) \cap \mathfrak{l}_{k}(\bar{x}, \overline{1+y})
$$

we see that

$$
\bar{\phi}(\overline{1+x+y})=\mathfrak{l}_{k^{\prime}}(\overline{1+\Phi(x)}, \overline{\Phi(y)}) \cap \mathfrak{l}_{k^{\prime}}(\overline{\Phi(x)}, \overline{1+\Phi(y)})
$$

while, on the other hand, a direct computation shows that

$$
\overline{1+\Phi(x)+\Phi(y)}=\mathfrak{l}_{k^{\prime}}(\overline{1+\Phi(x)}, \overline{\Phi(y)}) \cap \mathfrak{l}_{k^{\prime}}(\overline{\Phi(x)}, \overline{1+\Phi(y)}) .
$$

This forces

$$
\overline{1+\Phi(x+y)}=\bar{\phi}(\overline{1+x+y})=\overline{1+\Phi(x)+\Phi(y)}
$$

whence $\Phi(x+y)=\Phi(x)+\Phi(y)$.

Case 2: $1, \bar{x}, \bar{y}$ are all distinct in $F^{\times} / k^{\times}$(hence $1, \overline{\Phi(x)}, \overline{\Phi(y)}$ are all distinct in $F^{\prime \times} / k^{\prime \times}$; recall $\bar{\phi}$ is injective) but $1, \Phi(x), \Phi(y)$ are linearly dependent over $k^{\prime}$. Then, by assumption, there exists $z \in F^{\times}$such that $1, \Phi(x), \Phi(z)$ (equivalently $1, \Phi(y), \Phi(z)$ ) are linearly independent over $k^{\prime}$. Then, by the above, we have

(i) since $1, \Phi(x), \Phi(z)$ are linearly independent over $k^{\prime}$, we have

$$
\Phi(x+z)=\Phi(x)+\Phi(z)
$$

(ii) since $1, \Phi(x+y), \Phi(z)$ are linearly independent over $k^{\prime}$, we have

$$
\Phi(x+y+z)=\Phi(x+y)+\Phi(z)
$$

(iii) since $1, \Phi(x+z)=\Phi(x)+\Phi(z), \Phi(y)$ are linearly independent over $k^{\prime}$, we have

$$
\Phi(x+y+z)=\Phi(x+z)+\Phi(y)=\Phi(x)+\Phi(z)+\Phi(y) .
$$

Combining (ii), (iii), we obtain, again, $\Phi(\alpha x+\beta y)=\Phi(\alpha) \Phi(x)+\Phi(\beta) \Phi(y)$.

Case 3: $x=\alpha \in k^{\times}, y=x \in F^{\times} \backslash k^{\times}$. In 4.1.3 we established

$$
\Phi(\alpha+x)=\Phi(\alpha) \Phi\left(1+\alpha^{-1} x\right) \text { and } \Phi\left(\alpha^{-1} x\right)=\Phi(\alpha)^{-1} \Phi(x),
$$

so that it is enough to show that $\Phi(1+x)=1+\Phi(x)$. By assumption, there exists $y \in F$ such that $1, \Phi(x), \Phi(y)$ are linearly independent over $k^{\prime}$. In particular, by Case $1, \Phi(x+y)=\Phi(x)+\Phi(y)$. Then, the elements $1, \Phi(1+x), \Phi(y)$ are also linearly independent over $k^{\prime}$. Hence, by Case 1 , $\Phi(1+x+y)=\Phi(1+x)+\Phi(y)$. As a result,

$$
\overline{\Phi(1+x+y)}=\overline{1+\Phi(x+y)}=\overline{1+\Phi(x)+\Phi(y)}
$$

and

$$
\overline{\Phi(1+x+y)}=\overline{\Phi(1+x)+\Phi(y)}
$$

which forces $\Phi(1+x)=1+\Phi(x)$.

Case 4: $x=\alpha, y=\beta \in k^{\times}$. Let $x \in F^{\times} \backslash k^{\times}$. Then, on the one hand

$$
\Phi(1+\alpha x+\beta x) \stackrel{(1)}{=} \Phi(1+\alpha x)+\Phi(\beta x) \stackrel{(2)}{=} 1+\Phi(\alpha x)+\Phi(\beta x)=1+(\Phi(\alpha)+\Phi(\beta)) \Phi(x),
$$


where (1) is by Case 2 and (2) is by Case 3. While, on the other hand, if $\alpha+\beta \neq 0$, Case 3 also yields

$$
\Phi(1+\alpha x+\beta x)=1+\Phi((\alpha+\beta) x)=1+\Phi(\alpha+\beta) \Phi(x) .
$$

This shows $\Phi(\alpha+\beta)=\Phi(\alpha)+\Phi(\beta)$ unless $\beta=-\alpha$. For this last case, we have, by Case 3,

$$
\Phi(1+\alpha x)=1+\Phi(\alpha) \Phi(x), \Phi(1-\alpha x)=1+\Phi(-\alpha) \Phi(x)
$$

and, by Case 2,

$$
\Phi(2)=\Phi((1+\alpha x)+(1-\alpha x))=\Phi(1+\alpha x)+\Phi(1-\alpha x) .
$$

This implies

$$
2+(\Phi(\alpha)+\Phi(-\alpha)) x=\Phi(2) \in k^{\prime}
$$

hence $\Phi(\alpha)+\Phi(-\alpha)=0$.

Corollary 32. For every $x, y \in F^{\times}$we have

$$
\Phi(x y)=\Phi(x) \Phi(y) .
$$

Proof. By Lemma 31, we have

$$
\Phi(x(1+y))=\Phi(x)+\Phi(x y) \text { and } \Phi(1+y)=1+\Phi(y) .
$$

Also, since $\bar{\phi}: F^{\times} / k^{\times} \rightarrow F^{\prime \times} / k^{\prime \times}$ is a group morphism, we have

$$
\overline{\Phi(x y)}=\overline{\phi(x y)}=\bar{\phi}(\bar{x}) \bar{\phi}(\bar{y})=\overline{\Phi(x) \Phi(y)}
$$

and

$$
\begin{aligned}
\overline{\Phi(x)+\Phi(x y)}=\overline{\Phi(x(1+y))}=\overline{\phi(\overline{x(1+y)})}=\bar{\phi}(\bar{x}) \bar{\phi}(\overline{1+y}) & = \\
& =\overline{\Phi(x)(1+\Phi(y))}=\overline{\Phi(x)+\Phi(x) \Phi(y) .}
\end{aligned}
$$

This forces $\Phi(x y)=\Phi(x) \Phi(y)$ as claimed.

4.3. End of the proof. At this stage, we have shown that there exists a field morphism $\Phi: F \rightarrow$ $F^{\prime}$ such that

- $\Phi(k) \subset k^{\prime}$

- the induced group morphism $\bar{\Phi}: F^{\times} / k^{\times} \rightarrow F^{\prime \times} / k^{\prime \times}$ coincides with

$$
\bar{\phi}: F^{\times} / k^{\times} \rightarrow F^{\prime \times} / k^{\prime \times} \text {. }
$$

It remains to prove the unicity and Part (ii): $\Phi: F \rightarrow F^{\prime}$ is an isomorphism if $\bar{\phi}: F^{\times} / k^{\times} \rightarrow$ $F^{\prime \times} / k^{\prime \times}$ is.

4.3.1. Unicity. Let $\Psi: F \rightarrow F^{\prime}$ be another field morphism such that $\Psi(k) \subset k^{\prime}$ and $\bar{\Psi}=\bar{\phi}$. Then, for every $x \in F^{\times}$there exists a unique $\lambda_{x} \in k^{\prime \times}$ such that $\Phi(x)=\lambda_{x} \Psi(x)$. But necessarily we have $\lambda_{1}=1$ and, if $x \in F^{\times} \backslash k^{\times}$, so that $\Phi(x), \Psi(x) \in F^{\prime \times} \backslash k^{\prime \times}$, and

$$
1+\lambda_{x} \Psi(x)=1+\Phi(x)=\Phi(1+x)=\lambda_{1+x} \Psi(1+x)=\lambda_{1+x}(1+\Psi(x)) .
$$

This shows $\lambda_{x}=\lambda_{1+x}=1$. If $\alpha \in k^{\times}$and $x \in F^{\times} \backslash k^{\times}$, we have

$$
\lambda_{\alpha} \Psi(\alpha) \lambda_{x} \Psi(x)=\Phi(\alpha) \Phi(x)=\Phi(\alpha x)=\lambda_{\alpha x} \Psi(\alpha x) .
$$

Since $\lambda_{\alpha x}=\lambda_{x}=1$ by the above, this forces $\lambda_{\alpha}=1$ as well.

This concludes the proof of Lemma 29 (i). 
4.3.2. Part (ii). Lemma 29 (ii) follows formally from the existence and uniqueness assertion in Lemma 29 (i). Indeed, since $\phi^{-1}: F^{\prime \times} / k^{\prime \times} \rightarrow F^{\times} / k^{\times}$is also a group isomorphism which preserves lines, there exists a unique field morphism $\Psi: F^{\prime} \rightarrow F$ such that the induced group morphism $\bar{\Psi}: F^{\prime \times} / k^{\prime \times} \rightarrow F^{\times} / k^{\times}$coincides with $\phi^{-1}: F^{\prime \times} / k^{\prime \times} \rightarrow F^{\times} / k^{\times}$. Applying again this argument with the identity morphisms of $F^{\times} / k^{\times}, F^{\prime \times} / k^{\prime \times}$, one gets $\Phi \circ \Psi=I d_{F^{\prime}}, \Psi \circ \Phi=I d_{F}$.

\section{REFERENCES}

[1] E. Artin. Geometric algebra. Interscience Publishers, Inc., New York-London, 1957.

[2] H. Bass and J. Tate. The Milnor ring of a global field. Algebraic K-theory, II: "Classical" algebraic K-theory and connections with arithmetic (Proc. Conf., Seattle, Wash., Battelle Memorial Inst., 1972), pages 349-446. Lecture Notes in Math., Vol. 342, 1973.

[3] Fedor Bogomolov and Yuri Tschinkel. Milnor $K_{2}$ and field homomorphisms. In Surveys in differential geometry. Vol. XIII. Geometry, analysis, and algebraic geometry: forty years of the Journal of Differential Geometry, volume 13 of Surv. Differ. Geom., pages 223-244. Int. Press, Somerville, MA, 2009.

[4] Fedor Bogomolov and Yuri Tschinkel. Introduction to birational anabelian geometry. In Current developments in algebraic geometry, volume 59 of Math. Sci. Res. Inst. Publ., pages 17-63. Cambridge Univ. Press, Cambridge, 2012.

[5] François Charles and Bjorn Poonen. Bertini irreducibility theorems over finite fields. J. Amer. Math. Soc., 29:8194, 2016.

[6] Alexander Grothendieck. E.G.A. IV. Etude locale des schémas et des morphismes de schémas, troisième partie. Publ. Math. I.H.E.S., 28:5-255, 1966.

[7] Jean-Pierre Jouanolou. Théorèmes de Bertini et applications, volume 42 of Progress in Mathematics. Birkhäuser, 1983.

[8] Bruno Kahn. A sheaf-theoretic reformulation of the tate conjecture. 1998. arXiv:math/9801017.

[9] Serge Lang. On quasi algebraic closure. Ann. of Math. (2), 55:373-390, 1952.

[10] Serge Lang. Introduction to algebraic geometry. Interscience Publishers, Inc., New York-London, 1958.

[11] Hideyuki Matsumura. Commutative algebra. W. A. Benjamin, Inc., New York, 1970.

[12] A. S. Merkurjev and A. A. Suslin. K-cohomology of Severi-Brauer varieties and the norm residue homomorphism. Izv. Akad. Nauk SSSR Ser. Mat., 46(5):1011-1046, 1135-1136, 1982.

[13] Florian Pop. On the birational anabelian program initiated by Bogomolov I. Invent. Math., 187(3):511-533, 2012.

[14] Florian Pop. Recovering function fields from their decomposition graphs. In Number theory, analysis and geometry, pages 519-594. Springer, New York, 2012.

[15] John Tate. Symbols in arithmetic. Actes du Congrès International des Mathématiciens (Nice, 1970), Tome 1, pages 201-211, 1971.

[16] Adam Topaz. Reconstructing function fields from rational quotients of mod- $\ell$ Galois groups. Math. Ann., 366(12):337-385, 2016.

[17] Adam Topaz. A Torelli theorem for higher-dimensional function fields. 2017. arXiv:1705.01084.

[18] Vladimir Voevodsky. Motivic cohomology with Z/2-coefficients. Publ. Math. Inst. Hautes Études Sci., (98):59104, 2003.

[19] Vladimir Voevodsky. Reduced power operations in motivic cohomology. Publ. Math. Inst. Hautes Études Sci., (98):1-57, 2003.

Anna Cadoret

IMJ-PRG - SORBONnE UNIVERSITÉ, PARIS, FRANCE

anna.cadoret@imj-prg.fr

Alena Pirutka

CIMS, NeW York University, New York, U.S.A.

National Research University Higher School of Economics, RUSSiAn FEDERATiON

pirutka@cims.nyu.edu 